\title{
Effects of Matrix Heterogeneity on Animal Dispersal: From Individual Behavior to Metapopulation-Level Parameters
}

\author{
Eloy Revilla, ${ }^{1,2, *}$ Thorsten Wiegand,$^{1, \dagger}$ Francisco Palomares, ${ }^{2, \uparrow}$ Pablo Ferreras, ${ }^{3, \$}$ and Miguel Delibes ${ }^{2, \|}$
}

\author{
1. Department of Ecological Modeling, \\ Umweltforschungszentrum-Centre for Environmental Research, PF \\ 500136, D-04301 Leipzig, Germany; \\ 2. Department of Applied Biology, Estación Biológica de Doñana, \\ Spanish Council for Scientific Research-Consejo Superior de \\ Investigaciones Científicas, Avenida de Maria Luisa s/n, Pabellón \\ del Perú, E-41013 Sevilla, Spain; \\ 3. Instituto de Investigación en Recursos Cinegéticos, Consejo \\ Superior de Investigaciones Científicas-Universidad de Castilla La \\ Mancha-Junta de Comunidades de Castilla La Mancha, Apdo. \\ 535, E-13080 Ciudad Real, Spain
}

Submitted March 23, 2004; Accepted July 2, 2004;

Electronically published September 22, 2004 reproduced field estimates of population-level parameters (e.g., interpatch connectivity, maximum and final dispersal distances), and its performance was clearly improved when including the effect of matrix heterogeneity on movement properties. To assume there is a homogeneous matrix results in large errors in the estimate of interpatch connectivity, especially for close patches separated by open habitat or corridors of dispersal habitat, showing how important it is to consider matrix heterogeneity when it is present. Movement properties affect the interaction of dispersing individuals with the landscape and can be used as a mechanistic representation of dispersal at the metapopulation level. This is so when the effect of matrix heterogeneity on movement properties is evaluated under biologically meaningful spatial and temporal scales.

Keywords: autocorrelated random walk, Lynx pardinus, matrix fragmentation, individual-based spatially explicit simulations, interpatch connectivity, standard of plausibility.

Individual interpatch movement is a key process in the dynamics of spatially structured populations because it provides the linkage between the spatial subunits of the population and leads to the colonization of unoccupied areas (e.g., Pagel and Payne 1996; Brachet et al. 1999; Thomas 2000). In the extreme case of a metapopulation, separate subpopulations occupying suitable habitat patches undergo repeated extinction and recolonization events (Levins 1970). Long-term persistence of the metapopulation then arises from a balance between local extinction and recolonization through relatively infrequent interpatch dispersal movements. At the metapopulation level, these movement events can be described by interpatch connectivity, which is defined as the rate of migration between local populations (Moilanen and Hanski 2001). This metapopulation parameter is affected by properties of the focal and source patches (e.g., size, shape, quality), by properties of the donor and recipient populations (e.g., Allee effects, density dependence), and especially by the intervening habitat matrix, given that interpatch connectivity is the outcome of the interaction of behavioral processes with matrix heterogeneity during individual dispersal movements (e.g., Ferreras 2001; Ricketts 2001; Haynes and Cronin 2003).

One challenging aim of spatial ecology is generating
Am. Nat. 2004. Vol. 164, pp. E130-E153. (C) 2004 by The University of Chicago. 0003-0147/2004/16405-40364\$15.00. All rights reserved. 
models with some capacity to describe the interpatch movements at the metapopulation level. Island biogeography and metapopulation theory assume that space consists of patches of suitable habitat within a "matrix" of habitat unsuitable for the long-term maintenance of a population. The matrix is assumed to be homogeneous and sufficient to allow the passage of individuals. Under the assumption of matrix homogeneity, the use of simple models, including diffusion approaches, distance-dependent functions, and buffer measures, to summarize interpatch dispersal events has proved very useful (e.g., Hanski 1994, 1999; Turchin 1998; Cantrell and Cosner 1999; Higgins and Richardson 1999; Wiegand et al. 1999; Vos et al. 2001; Moilanen and Nieminen 2002). These functional forms are appealingly simple, and their structure and associated parameters represent migration at the scale of the metapopulation.

However, mounting theoretical and empirical evidence shows that variations in matrix quality can have a wide range of effects on metapopulation dynamics (Vandermeer and Carvajal 2001). This is so because different matrix types contribute significantly to patch isolation in nontrivial ways (e.g., Norton et al. 2000; Perault and Lomolino 2000; Ferreras 2001; Ricketts 2001; Vandermeer and Carvajal 2001), indicating the need to incorporate a behavioral component of matrix use into models of interpatch connectivity. Therefore, when modeling interpatch connectivity, we have to test whether the assumption of matrix homogeneity is valid and, if not, screen alternative models.

As the impact of matrix quality comes through effects on individual behavior and then translates into higher spatiotemporal scales (such as interpatch connectivity), the information obtained on the individual scale can be directly used to generate individual-based models. These models can provide a powerful tool for analyzing the impact of matrix structure on long-distance interpatch movements and on (meta)population dynamics because they allow for the inclusion of behavioral rules on the response of individuals to the landscape, potentially linking individuals' use of space (dispersal and habitat selection) to population and metapopulation phenomena (Pulliam and Dunning 1995; Lima and Zollner 1996; Wiegand et al. 1999). This approach means linking two or more spatiotemporal scales and demanding a mechanistic representation of the process in which the important details necessary to reproduce the pattern are included (Levin 1992; Wiegand et al. 2003). However, there is a general concern about the use of individual-based spatially explicit models because of two main arguments.

First, it is inherently difficult to obtain information on the actual behavior of individuals moving in the matrix during interpatch long-distance dispersal events (Nathan et al. 2003). At the level of individuals, much recent work has improved our understanding of the effect of individual behavior on movement properties (e.g., With 1994; Haddad 1999a, 1999b; Haddad and Baum 1999; Kindvall 1999; McIntyre and Wiens 1999; With et al. 1999). This work is mostly in relation to corridors and stepping-stones of natural breeding habitat (i.e., the habitat that forms the patches) using controlled experimental settings in which animals are released under different environmental conditions. Individual behavior is quantified as distributions of step lengths, turning angles, and edge-avoidance behavior, all of which may be used to parameterize correlated random-walk models (Lagrangian description of the movement; Turchin 1998; Nathan et al. 2003). However, it is not clear how the results of these experimental studies apply to animals that have complex cognitive capacities and that perform extensive movements in the matrix, especially during natal or breeding dispersal, when the movement is triggered by different behavioral cues than when individuals are translocated or when they are foraging (Koenig et al. 1996; Linnell et al. 1997; Fisher and Lindenmayer 2000; Ricketts 2001; note the different meaning of dispersal in population biology and in the description of life-history traits used in behavioral ecology, e.g., interpatch dispersal vs. natal dispersal). In summary, our knowledge of individual movement behavior in heterogeneous matrices during interpatch dispersal events is still very poor (Ricketts 2001).

Second, by analyzing movement paths, we can obtain many potential movement rules in terms of individual behavior. However, the fact that movement properties depend on the spatial and temporal scales and on life-history traits that may change during the life of individuals (e.g., With 1994; Turchin 1996) makes suspicious the use of movement rules obtained at local scales to predict parameters at the population scale without a prior evaluation. In individual-based dispersal models, we assume a mechanistic relationship between model structure and the real behavioral processes involved in animal movement in the hope that we can reproduce the parameters of interest at the level of the population (e.g., interpatch connectivities or dispersal distance and mortalities; Mooij and DeAngelis 2003; Wiegand et al. 2003). However, the lack of validation of the mechanistic assumption of "too complex" individual-based dispersal models has cooled the initial enthusiasm (Pulliam and Dunning 1995) about their use (e.g., Doak and Mills 1994; Ruckelshaus et al. 1997; Beissinger and Westphal 1998; Hartway et al. 1998).

In this study, we first adopt an approach based on individual behavior to determine how matrix heterogeneity affects the properties of individual movements at large spatial scales using telemetric data on the interpatch movements performed by individual Iberian lynx (Lynx par- 
dinus) during their natal dispersal in a metapopulation at two temporal scales: hourly (intraday) and daily (interday) radiolocations. Note that in this case, interpatch movements occur only during individual natal dispersal. Second, using the movement properties at the intraday scale where the interaction of individuals with matrix structure occurs, we develop an individual-based spatially explicit simulation model to analyze, over broad ranges of parameter values, which types of movement rules chiefly affect model predictions at higher scales (i.e., the individual [between days] and metapopulation [interpatch connectivity] scales). Third, we parameterize this simulation model using the information obtained in the analysis of field data and evaluate whether the model is able to reproduce, at the population scale, field estimates of parameters such as interpatch connectivities or dispersal distances. Fourth, to evaluate the importance of the assumption of a homogeneous matrix in the case of the Iberian lynx metapopulation, we investigate how the impact of matrix heterogeneity on movement rules translates into the values of interpatch connectivity by comparing the results of the fully specified simulation model with the results of the same model without considering matrix heterogeneity (i.e., only distance dependent) and with the field estimates of interpatch connectivities.

\section{Methods}

The Site, the Species, and the Metapopulation

The study was carried out in Doñana $\left(\sim 2,500 \mathrm{~km}^{2}\right.$ in southwestern Spain; fig. 1), a flat region where croplands cover $49 \%$ of the area. The remaining highly fragmented vegetation is dominated by Mediterranean scrubland ( $\mathrm{Hal}-$ imium spp., Cistus spp., and Pistacia lentiscus), pine (Pinus pinea) and eucalyptus (Eucalyptus sp.) plantations, and marshlands. In the south of this area is located Doñana National Park, covering $\sim 550 \mathrm{~km}^{2}$ (fig. 1).

The Iberian lynx, Lynx pardinus, is considered the most endangered wildcat in the world (Delibes et al. 2000). It inhabits only the southern parts of the Iberian Peninsula, distributed in two isolated populations, each of which has a metapopulation structure (Gaona et al. 1998; Palomares et al. 2003). Habitat degradation and fragmentation are the main threats for the conservation of the Iberian lynx (Delibes et al. 2000). As for many other species, its future survival depends on our ability to understand the dynamics of its metapopulations in the context of landscape design, management, and development, which in turn depend on how habitat features and landscape patterns promote and/or constrain dispersal between subpopulations (Gaona et al. 1998; Ferreras et al. 2001). Resident lynx are territorial (Palomares et al. 2001). The Doñana metapopulation is close to carrying capacity and the total number of suitable territories is low (10-14 territories). These territories are located in isolated patches of wellpreserved Mediterranean scrubland that form eight subpopulations (fig. 1; Gaona et al. 1998; Ferreras et al. 2001). Individuals disperse from their natal areas when between 8 and 23 months old (Ferreras et al. 2004). Natal dispersal can last between a few weeks and $>1$ year.

\section{Field Methods}

Lynx were captured and radio-tagged in two of the source populations included inside Doñana National Park: Reserva Biológica $(r b)$ and Coto del Rey ( $c r$; fig. 1; for more details, see Palomares et al. 2000, 2001). Between 1984 and 1991, we marked a total of 12 lynx that dispersed from Reserva Biológica and, between 1991 and 1999, 15 animals that dispersed from Coto del Rey (fig. 1). Additionally, we marked three dispersing individuals whose population of origin was unknown (already dispersing when trapped). For the purpose of analyzing dispersal movements in the matrix, natal dispersal was considered to begin when individuals left the natal subpopulation and to end when they settled or died or when the radio transmitter failed (Palomares et al. 2000; Ferreras 2001). Settlement was determined by measuring site fidelity over short time intervals through computer simulations (for a detailed description of the analytical methodology used to determine the onset and the end of dispersal, see Palomares et al. 2000). These 30 animals were tracked during their dispersal period in the matrix for an average of $115 \pm 28.1$ days $( \pm 1$ $\mathrm{SE})$.

Dispersing lynx were radiolocated via triangulation from two bearings, normally at $<1 \mathrm{~km}$ from the animal to decrease triangulation error (determined in a ground test as $<207 \mathrm{~m}$ in $95 \%$ of cases). During the last 4 years of the study, animals were frequently located by homing to the actual position using a global positioning system (Garmin $75)$. The global positioning system error was estimated in the field during the period of study $(<100 \mathrm{~m}$ in $97 \%$ of the cases). We searched the entire Doñana area by aircraft when we lost radio contact with lynx. We defined periods of daily activity (when lynx actually move) between two consecutive resting points, usually lasting between 8 and 24 consecutive hours during part of the sunset, night, and sunrise. In total, we monitored 57 periods of activity with a sampling frequency of one location per hour. Additionally, we obtained $44 \pm 11.6$ locations per individual (range, three to 312 locations) with a sampling frequency of one location per day. 

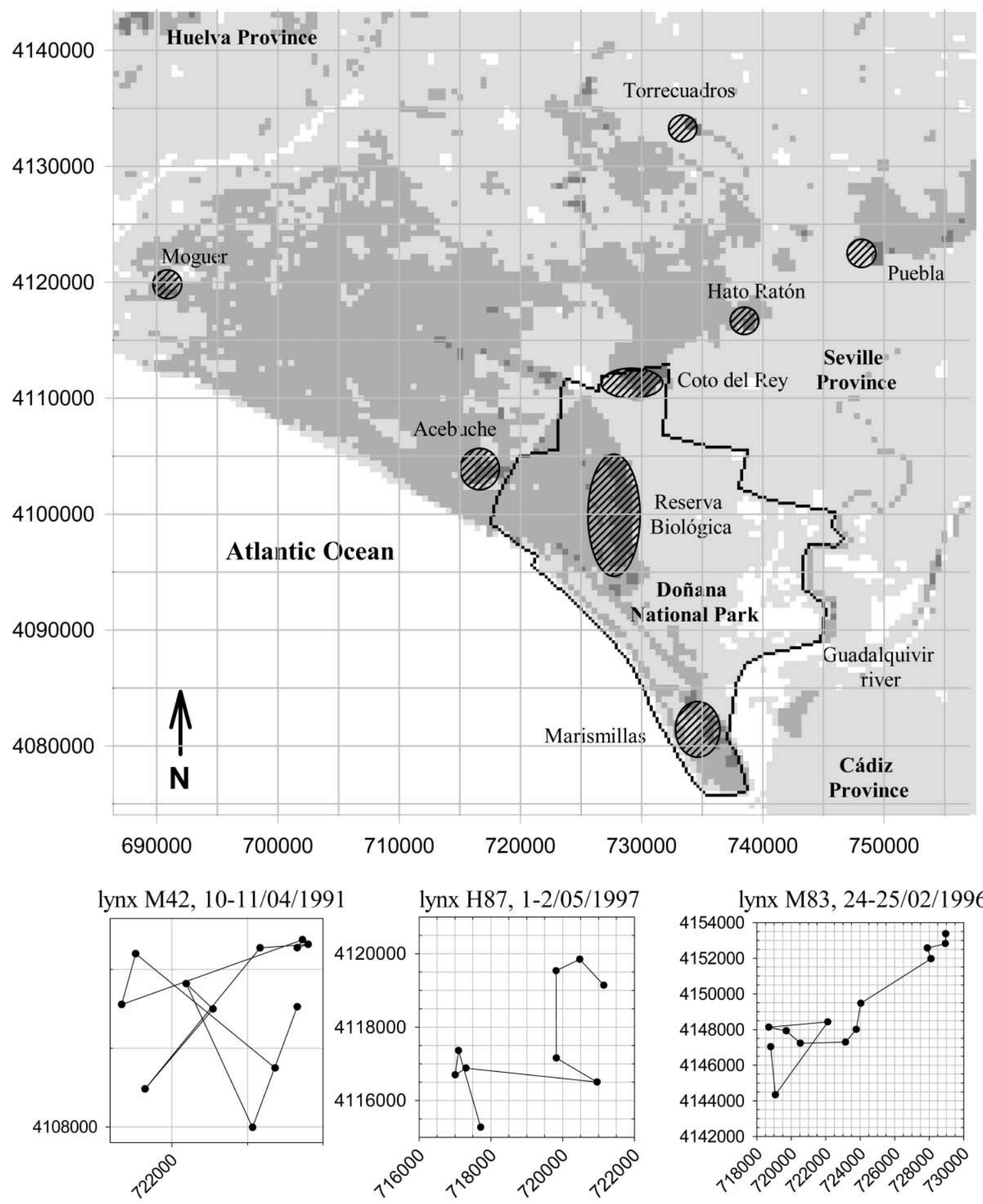

Figure 1: Top, Landscape map of the study area showing the habitat types barrier (white), open habitat matrix (light gray), dispersal habitat matrix (gray), and the breeding habitat (dark gray) of the eight subpopulations (crossed ellipses) forming Doñana metapopulation of Iberian lynx. The limits of the Doñana National park are shown as a solid black line. The spatial grain of the landscape map is $500 \mathrm{~m} \times 500 \mathrm{~m}$. The radio-tagged dispersing lynx were trapped at the Reserva Biológica and Coto del Rey subpopulations. Reference system is Universal Transverse Mercator (i.e., one grid cell of the reference system refers to 5,000 $\mathrm{m} \times 5,000 \mathrm{~m}$ ). Bottom, Examples of movement paths of dispersing Iberian lynx during one period of activity. Note the great variety in the spatial scale (the grid represents cells of $500 \mathrm{~m} \times 500 \mathrm{~m}$ ). 


\section{Lynx Habitat Selection in the Matrix and the Spatial and Temporal Scales}

From a metapopulation perspective the patches forming subpopulations are defined after lynx breeding habitat (fig. 1; Gaona et al. 1998; Ferreras et al. 2001; Fernández et al. 2003; Palomares et al. 2003), characterized by wellpreserved Mediterranean scrubland where resident lynx establish their territories (Palomares et al. 2000, 2001; Palomares 2001). The matrix in which breeding habitat patches are embedded is formed by any other land use types that the animals utilize during their interpatch movements. Previous work on lynx habitat selection have shown that this matrix is heterogeneous and has to be divided into two types of habitat (Palomares et al. 2000, 2001; Palomares 2001): open habitat, which is avoided but still used, and dispersal habitat, which is used as available. Open habitat is characterized by land use types with almost no protective cover (open vegetation such as marshland, dunes, cropland, or pastureland), while dispersal habitat offers protective cover (mostly forested areas; Palomares et al. 2000). Additionally, urban areas and wide water bodies form barrier habitat, which dispersing lynx never use in their movements. We assigned the types of habitat to animal locations using IDRISI Geographical Information System (Eastman 1995) based on a 1997 reclassified land cover map of the area (Sistema de Información Ambiental de Andalucía; Moreira and Fernández-Palacios 1995; Palomares et al. 2000). At the spatial resolution and for the habitat types that we consider, the landscape has not changed significantly during the period of study, except for one area that was transformed into croplands (dispersal habitat was converted into open habitat). In the description of movement properties accounting for matrix heterogeneity, we did not consider data obtained from from one animal when it used that area in 1984 (before the transformation) because our landscape map reflects the habitat distribution in 1997 (hence, sample sizes may not be coincident between different analyses).

To define the spatial resolution of the analyses, we first considered the triangulation error of the telemetric data $(<0.2 \mathrm{~km}$ in $95 \%$ of locations $)$. Second, we used the hourly tracking data and analyzed the distribution of distances moved during $1 \mathrm{hr}$ without accounting for the underlying landscape. The average distance moved per hour was $0.7 \mathrm{~km}$ (see "Results"). Roughly $80 \%$ of all steps were $<1 \mathrm{~km}$, and $57 \%$ were $<0.5 \mathrm{~km}$. Thus, in order to avoid losing substantial detail on dispersal distances, we needed a spatial resolution $<1 \mathrm{~km}$. Finally, we visualized the map of the four habitat categories (breeding, dispersal, open, and barrier habitats) at different cell sizes between $0.2 \mathrm{~km}$ (the error of the telemetric data) and 1 $\mathrm{km}$ and found that important spatial structures disap- peared above a cell size of $0.5 \mathrm{~km}$. Therefore we selected a cell size of $0.5 \mathrm{~km} \times 0.5 \mathrm{~km}$ as the grain of the landscape map (fig. 1).

We consider two different temporal scales with a straightforward biological meaning. The interaction of dispersing lynx with the landscape occurs while they move during their periods of daily activity. Therefore we defined intraday movement steps (within one daily activity period) as those between two consecutive radiolocations separated by $1 \mathrm{hr}$ during the same period of activity. On a coarser temporal scale, we defined one daily movement step between two locations recorded in consecutive days (usually between two resting points). Finally, we also considered the temporal scale defined by the duration of the dispersal event (see "Modeling Strategy and Model Analysis"). The two upper temporal scales should reflect the outcome of the interaction of the animals with the landscape.

\section{Analysis of Dispersal Movements}

First, we analyze the properties of the movement during the period of activity (intraday analyses) independently of the underlying matrix. Then we describe the effect of the different types of matrix (open and dispersal habitats) on movement properties. Finally, we repeat the same analyses for the daily time steps (daily analyses, between two consecutive resting points). In all these analyses, we used the assumptions of a random walk as null model.

Description of movement properties. We considered the following movement properties: distances, defined as the euclidean distance between two consecutive radiolocations, and turning angles, defined as the change in direction between two consecutive movement vectors (i.e., three consecutive radiolocations define one turning angle). For each period of activity, we calculated the euclidean distances moved per hour, the euclidean distance between the first and the last location (net distance), and the total distance moved during the period of activity (sum of the distances of all movement vectors). At the daily level, distance moved was the Euclidean distance between two locations of consecutive days.

We analyzed the effect of the interface between dispersal and open habitats on movement directionality by comparing the distribution of turning angles under different availabilities of dispersal habitat surrounding the second radiolocation defining the angle (i.e., where the animal makes the turning decision). Data were grouped in different classes of availability when the sample size was too small. After graphical inspection of the distribution of turning angles (in order to assess unimodality), we tested for departures from an autocorrelated random walk using Rayleigh $V$-test, that is, for a null hypothesis considering a mean angle oriented toward $0^{\circ}$ (i.e., moving straight 
Table 1: Values of model parameters explored in the sensitivity analysis and their corresponding field estimates

\begin{tabular}{|c|c|c|c|c|c|c|c|c|c|}
\hline Parameter & $\bar{\phi}_{d}$ & $\theta_{d}$ & $N_{d}$ & $\beta$ & $\gamma$ & $L$ & $\Delta \theta_{l}$ & $\Delta \theta_{f}$ & $\delta$ \\
\hline $\begin{array}{l}\text { Range explored } \\
\text { Field estimate }\end{array}$ & $12.4-25.4$ & $.0-.4$ & $2-8$ & $.25-1.00$ & $.035-.095$ & $2-8$ & $.15-.45$ & $.00-.45$ & $.49-1.00$ \\
\hline ( \pm uncertainty) & $18.9(.5)^{\mathrm{a}}$ & $.26(.034)^{\mathrm{b}}$ & $5(4-5)^{\mathrm{b}}$ & $.8(.15)^{\mathrm{a}}$ & $.09(.002)^{\mathrm{c}}$ & $6(4-8)^{a}$ & $.27(.02)^{\mathrm{b}}$ & $.10(.06)^{\mathrm{b}}$ & $.65(.14)^{\mathrm{b}}$ \\
\hline
\end{tabular}

Note: $\bar{\phi}_{d}=$ average number of steps moved per day; $\theta_{d}=$ movement autocorrelation in dispersal habitat; $N_{d}=$ number of cells of dispersal habitat defining fragmentation; $\beta=$ avoidance of the matrix; $\gamma=$ probability to return to dispersal habitat; $L=$ long-distance movements; $\Delta \theta_{l}=$ increase in movement autocorrelation in long-distance movements; $\Delta \theta_{f}=$ increase in movement autocorrelation in fragmented areas; $\delta=$ bimodal distribution of turning angles in dispersal habitat.

a SE based on the regression parameter.

${ }^{b}$ SE based on individual variation.

c $5 \%$ variation around the field value.

forward; Batschelet 1981; Zar 1999), as well as in the cases in which we had reasons to expect an orientation toward $180^{\circ}$ (i.e., turning back). When our null hypothesis was a fully random walk, we used Rayleigh $z$-test (Batschelet 1981; Zar 1999). We also examined data for correlation between consecutive turning angles using parametric angular-angular correlation tests, $r_{\text {aa }}$ (Zar 1999), and determining significance by randomizing (10,000 times) the data set, recalculating $r_{\text {aa }}$, and then obtaining the 95\% probability distribution of the statistic for the null hypothesis of no correlation. We calculated correlation between consecutive daily distances and its significance using an analogous randomization procedure (also 10,000 times). We analyzed the effect of sex, season, days since the beginning of dispersal, and the individual (nested in the model to control for individual variability) on movement properties using general linear models (Type III sum of squares, PROC GLM; SAS Institute, Cary, N.C.). Data were transformed when necessary to produce normality and homogeneity of variances.

\section{Modeling Strategy and Model Analysis}

We aimed to investigate whether we can attain predictive capacity at the population level based on movement rules derived from movement properties at the individual level. With this purpose, we created an individual-based spatially explicit simulation model (full model) based on the movement properties at the intraday temporal scale (during one period of activity). The simulation model is constructed with data from the intraday temporal scale, while model predictions represent the system at higher scales (individual scale at a daily temporal resolution and population scale at a temporal resolution of dispersal event). For effective model analysis, we followed the field protocol as closely as possible; we simulated the same number of animals released at the same locations as they started their dispersal in the field (Reserva and Coto del Rey populations or capture sites when the population of origin was unknown) during the same number of days and using the same landscape for model simulations as used to describe the movement properties. With this setting, we aimed to remove the effect that demography (mostly mortality of both dispersing and resident animals) might have on model predictions and hence make these model predictions directly comparable with field data.

During the simulations, we collected data following the same protocol as with field data, deriving predictions at the individual scale (daily, between two resting points) and population scale (dispersal event) for which we had field estimates. We considered the following model predictions as patterns to evaluate model performance (Wiegand et al. 2003): hab, the proportion of locations in open habitat matrix at the daily temporal resolution; netday, average net distance moved between days; $a v d_{30}$ and $a v d_{120}$, average distance to the origin of dispersal at days 30 and $120 ; r b$ $c r, r b-m a, r b-a c, r b-h a, r b-t o, r b-p u$, and $r b-m o$, connectivities between the Reserva Biologica subpopulation and Coto del Rey, Marismillas, Acebuche, Hato Raton, Torrecuadros, Puebla, and Moguer subpopulations (fig. 1); $\mathrm{cr}$ $r b, c r-m a, c r-a c, c r-h a, c r-t o, c r-p u$, and $c r-m o$, connectivities between the Coto del Rey subpopulation and Reserva Biologica, Marismillas, Acebuche, Hato Raton, Torrecuadros, Puebla, and Moguer subpopulations (fig. 1); $m r b$ and $m c r$, average of the maximum dispersal distances (net distance between the origin and the furthest point reached by each individual) of animals beginning dispersal from Reserva Biologica and Coto del Rey subpopulations, respectively; and erb and ecr, average of the final dispersal distances (net distance between the origin and the final point reached by each individual) of animals beginning dispersal from Reserva Biologica and Coto del Rey subpopulations, respectively.

Model sensitivity. We performed a sensitivity analysis over a biologically plausible range of parameter values to determine which parameters of the simulation model (i.e., movement rules) had the largest impacts on model predictions. We created a total of 25,000 random model parameterizations (from uniform distributions within the ranges given in table 1). For each model parameterization, 
we performed 50 replicate simulations. Note that we are interested in evaluating the model over biologically meaningful parameter ranges and not in assessing the role of parameter uncertainty (although the impact of parameter uncertainty can be directly approximated from the regressions of the sensitivity analysis).

To investigate the sensitivity of model output to the different parameters, we performed multiple linear regressions with a model prediction (the average of the 50 replicates) as dependent variable and model parameters as independent variables (McCarthy et al. 1995). The resulting equation approximates the functions that relate the parameters of the simulation model to predictions in a simple way. We did not consider interactions between parameters because the regression models with only main effects explained most of the variance observed in the predictions (see "Results"). We scaled the dependent and independent variables to values between 0 and 1 to standardize the coefficients of regression models for different predictions. We used these standardized coefficients, $S_{i}$, as indices that describe the sensitivity of the model output to the parameters $p_{i}$. Therefore, $S_{i}$ values close to 1 indicate strong sensitivity while parameters in the simulation model with $S_{i}$ values close to 0 have little impact on a given model prediction $i$.

Model performance and matrix heterogeneity. We investigated the capacity of the full model, parameterized with observed field data, to reproduce the values observed in the field for the predictions described above (field values for the predictions were estimated from the same dataset of dispersing individuals; further details are given in Palomares et al. 2000; Ferreras 2001; Ferreras et al. 2004; see "Results"). Additionally we created a simplified simulation model (basic model) with the same structure as the full model but without the rules on habitat selection in the matrix, thus assuming a homogeneous matrix in which dispersal and open habitats are the same. The basic model was also parameterized with field data (i.e., both models used the same parameter values for their common rules). We compared the performance of both models using a multivariate analysis of variance (MANOVA) in which all dependent variables (model predictions) were included in a single analysis to reduce the risk of Type I errors associated with multiple comparisons. We used the type of model as the independent variable. In order to include most of the variability in the predictions, we used 1,000 simulations from each model parameterized with the field estimation of the parameters (hence $n=2,000$ ). Then we performed the univariate test for each of the predictions (MANOVA analysis; PROC GLM in SAS). We also calculated the probability of obtaining the values of the predictions observed in the field (i.e., patterns) by calculating the corresponding $z$-scores from the probability distri- butions obtained with the basic and full models (from the same 1,000 simulations).

\section{Results \\ Intraday Analyses without Accounting for Matrix Heterogeneity}

In this section, we analyze the data set on the 57 periods of lynx monitoring during one period of activity (with a sampling frequency of one location per hour) without accounting for the underlying habitat types.

Net distance, total distance, and distance moved per hour. Net distance (from the first to the last location) moved by lynx was up to a maximum of $12.2 \mathrm{~km}$ while the total distance ranged from 0.3 to $38.7 \mathrm{~km}$ (table 2; the difference between their means being significant, Wilcoxon signed rank test, $W=1450, P<.001)$. These broad ranges indicate that within one period of activity there was a large variation in the scale of movement (fig. 2). Net daily distance was correlated with total daily distance (both log transformed, $r^{2}=0.40, F=36.9$, df $\left.=1,55, P<.001\right)$. Lynx returned to the proximity of the initial position (considered as when net distance $<0.5 \mathrm{~km}$ ) in $14 \%$ of the periods of activity, in these cases moving on average $7.2 \pm 5.8 \mathrm{~km}$. Thus, during one period of activity, lynx may perform extensive movements that would be undetected if we only sample at daily intervals. There was no effect of sex, season, time since the beginning of dispersal, individual, or the number of locations on the net distance moved (log transformed, $F=0.41$, df $=22,34, P=$ .9848 ), while total daily distance was affected only by the number of locations (i.e., by the length of the period of activity, $F=8.93$, df $=1,34, P=.0053)$. The distance moved per hour ranged between 0 and $10.2 \mathrm{~km}$. The general linear model for the distance moved per hour (log transformed, $F=2.61$, df $=58,701, P<.0001)$ showed that only the period of activity was significant-that is, lynx moved consistently faster during the same periods of activity $(F=2.16, \mathrm{df}=52,701, P<.0001)$-while season, sex, time since the beginning of dispersal, and individuals were not $(F<3.27, P>.071$ in all cases $)$. The inverse cumulative probability distributions of net and total distances and of distances moved per hour follow exponential decay functions (table 2).

Turning angles. At the hourly temporal scale, the distribution of turning angles was different from random (Rayleigh $z=9.28, P<.001, n=451$ ), being oriented toward $0^{\circ}$ (Rayleigh $V$-test, $u=4.25, P<.0005, n=451$ ). By analyzing two consecutive movement steps we found a very weak but significant correlation between the cosine of the turning angle $\cos (t)$, and the logarithm of the distance $d_{2}$ moved in the second step $\left(r=0.24, r^{2}=0.06\right.$, 
Table 2: Distribution of observed dispersal distances $d(\mathrm{~km})$ for different temporal resolutions and types of matrix habitat (dispersal and open habitats)

\begin{tabular}{|c|c|c|c|c|c|c|}
\hline \multirow[b]{2}{*}{ Dispersal distances } & \multirow[b]{2}{*}{ Mean \pm SD } & \multicolumn{2}{|c|}{ Parameters } & \multicolumn{3}{|c|}{ Significance } \\
\hline & & $a$ & $b$ & $r^{2}$ & $P$ & $n$ \\
\hline \multicolumn{7}{|l|}{ Intraday: } \\
\hline Net distance per day & $3.43 \pm 3.09$ & .993 & .137 & .99 & $<.00001$ & 57 \\
\hline Total distance per day & $9.54 \pm 6.65$ & .00027 & $\ldots$ & .99 & $<.00001$ & 57 \\
\hline \multicolumn{7}{|l|}{ Distance moved per hour: } \\
\hline Total & $.70 \pm 1.16$ & .860 & .740 & .98 & $<.0001$ & 760 \\
\hline Inside dispersal habitat & $.63 \pm .98$ & .866 & .756 & .98 & $<.0001$ & 344 \\
\hline Inside open habitat & $.58 \pm .71$ & .860 & .749 & .98 & $<.0001$ & 146 \\
\hline Mixed movements & $1.82 \pm 2.35$ & 1.084 & .383 & .98 & $<.0001$ & 76 \\
\hline \multicolumn{7}{|l|}{ Interday: } \\
\hline Total & $2.43 \pm 2.28$ & 1.464 & .212 & .99 & $<.00001$ & 691 \\
\hline Inside dispe & $2.42 \pm 2.26$ & 1.046 & .212 & .99 & $<.00001$ & 529 \\
\hline Females & $1.76 \pm 1.49$ & 1.084 & .292 & .99 & $<.00001$ & 189 \\
\hline Males & $2.78 \pm 2.52$ & 1.040 & .179 & .99 & $<.00001$ & 340 \\
\hline Inside open habitat & $3.88 \pm 2.87$ & 1.093 & .121 & .98 & $<.0001$ & 27 \\
\hline Mixed movements & $2.17 \pm 2.13$ & 1.068 & .249 & .99 & $<.00001$ & 139 \\
\hline
\end{tabular}

Note: Data were fitted to the general exponential model $p(d)=a \exp (-b d)$, with $p$ being the frequency of distances $d$. In the case of the total distance per day, the data followed the function $p(d)=\left(1+a d^{3}\right)^{-1} \cdot n=$ sample size.

$F=32.67, \mathrm{df}=1,537, P<.01 ;$ fig. 2). To investigate whether movements forward (positive cosine) and backward (negative cosine) depended on the distance moved in the second step, we used these two categories as dependent variable in a logistic regression using the log distance moved as predictor $\left(-2 \log L \chi^{2}=13.33, \mathrm{df}=1\right.$, $P=.0003$; classifications $52.8 \%$ concordant, $46.1 \%$ discordant, and $1 \%$ tied). We used this logistic model to find the threshold above which the animals had a larger probability of moving forward $(3.0 \mathrm{~km}$, predicted probability $>0.5$ ). When animals moved steps larger than this threshold value, the average cosine was $0.47 \pm 0.19$ (vs. $0.18 \pm 0.03, \pm 1 \mathrm{SE}$, for distances $<3.0 \mathrm{~km}$; fig. 2). The low number of movements $>3.0 \mathrm{~km}$ explains why the relation between the cosine of the turning angle and $\log \left(d_{2}\right)$ was so weak (fig. 2).

There was no correlation between two consecutive turning angles (angular-angular correlation $r_{\mathrm{aa}}=0.006, n=$ 348 , random $95 \%$ confidence interval $[\mathrm{CI}]=-0.007$ to 0.007). We did not detect any effect of sex, season, time since the beginning of dispersal, and individuals on $\cos (t)$ $(F=1.38, \mathrm{df}=24,426, P=.1128)$. We obtained the same results with a model including a dummy variable to identify the period of activity $(F=1.13, \mathrm{df}=43,407$, $P=.2669$ ).

\section{Intraday Analysis Accounting for Matrix Heterogeneity}

In this section, we analyze the same data set as in the previous section, but now we consider the effect of the underlying types of matrix habitat on movement properties.

Distance moved per hour. Lynx displacements in mixed movements (i.e., one of the locations is in dispersal and the other in open habitat) were longer than movements in open or in dispersal habitats (Kruskal-Wallis ANOVA on ranks, $H=48.71, \mathrm{df}=2, P<.001$; Dunn's post hoc test, $Q>6.10, P<.05$ in comparisons including mixed movements), probably indicating that lynx move faster to avoid the proximity of open habitat. The inverse cumulative probability distributions of distances moved per hour in different types of matrix followed similar exponential decay functions (table 2). There was no correlation between the number of cells of dispersal habitat surrounding the first location of the animal (where the individual takes the movement decision) and the distance moved in the next hour $(r=0.02, P=.7)$.

Probability to move to and stay in open habitat. During one period of activity, lynx moved from dispersal habitat to open habitat on $20.2 \%$ of the displacements. In cases when the animals were surrounded by five or more cells of dispersal habitat, the probability to move to cells of open habitat was similar to the probability expected for a random choice of the cell (fig. $3 A$ ). However, when the number of cells of dispersal habitat surrounding the animal was lower, the probability to move to open habitat was greatly reduced (fig. $3 A$ ). In general, lynx spent little time in open habitat, and it was rare when they remained in it at the end of the period of activity (fig. $3 B$ ). The probability of staying in open habitat as a function of the 



Figure 2: $A$, Relationship between the distance $\left(d_{2}\right)$ moved during the second vector of two consecutive hourly steps (log transformed) and the turning angle $\alpha . B$, Distribution of the percentages of turning angles (grouped by cosine intervals) when the distance moved in the second vector that defines the angle $\left(d_{2}\right)$ is larger or smaller than $3,000 \mathrm{~m}$ for the hourly steps. Positive cosines represent movements forward; negative cosines represent movements backward.

time spent on it followed an exponential decay, with only $4.8 \%$ of the cases lasting $>5 \mathrm{hr}$ in the matrix (fig. $3 B$ ).

Matrix effects on turning angles. To analyze the effect of the type of matrix on turning angles, we distinguished between turning angles in which the second position of the animal (i.e., where it decides the direction of the movement) was in dispersal habitat and those that were in open habitat.

In cases when the second position of the animal was located in dispersal habitat surrounded by seven or eight cells of dispersal habitat, the distribution of turning angles was bimodal (fig. 4A). This bimodal distribution lay along a diameter line oriented at the axis $0^{\circ}-180^{\circ}$ (Rayleigh $V$ - test, $u=4.98, P<.0005$ ), with $27 \%$ forward movements and $17 \%$ backward movements (it should be $12.5 \%$ under a uniform distribution; fig. $4 A$ ). In the rest of the cases, the distributions of angles were not bimodal (fig. 4). When the animal was surrounded by three to six cells of dispersal habitat, the turning angles were significantly oriented toward $0^{\circ}$ (Rayleigh $V$-test, $u=4.54, P<.0005$ ), with $31 \%$ of movements oriented forward (fig. $4 B$ ). However, when there were fewer cells of dispersal habitat around the position of the animal (i.e., seven or more cells of open habitat), the movement was not different from random (Rayleigh $z=.69, P>.25$; fig. $4 C$ ).

In the second case, when the animal was in open habitat 

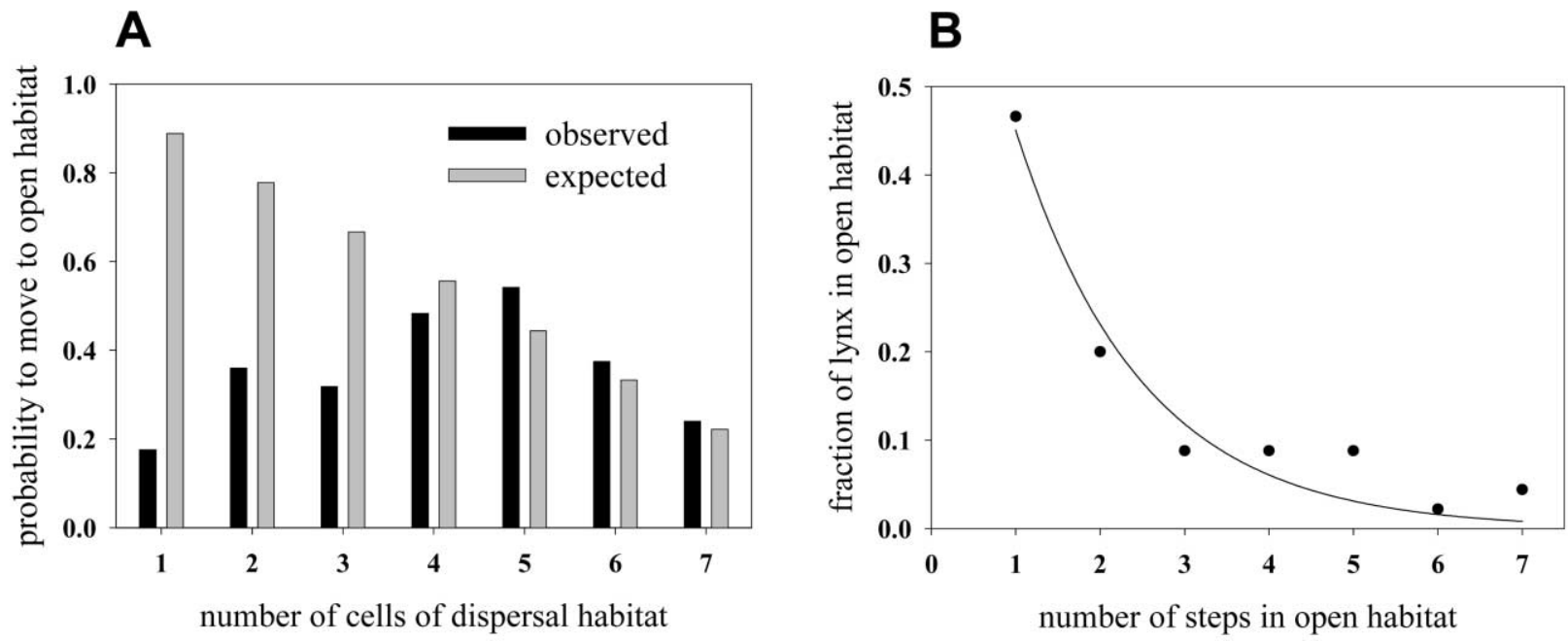

Figure 3: A, Observed (black bars) and expected (gray bars) probabilities to move from a cell of dispersal habitat to a cell of open habitat as a function of the number of cells of dispersal habitat surrounding the animal. Expected probability is based on a random movement. $B$, Probability to stay in open habitat, as a function of the number of steps (i.e., hours) moved in open habitat. The function follows an exponential decay with the form $P(s)=0.879 \exp (-0.669 s)$, where $P(s)$ is the probability and $s$ is the number of steps of $1 \mathrm{hr}$ spent in open habitat $\left(r^{2}=0.95, F=90\right.$, $\mathrm{df}=1,5, P=.0002)$. Both refer to movements during one period of activity.

and surrounded by four or more or from one to three cells of dispersal habitat, the movement was significantly oriented toward $0^{\circ}$ (Rayleigh $V$-test, $u=2.19, P<.02$, and $u=2.13, P<.02$, respectively), with $36 \%$ and $30 \%$ of forward-turning angles (fig. $4 D, 4 E$ ). When there were no cells of dispersal habitat surrounding the animal, the movement was not different from random (Rayleigh $\mathrm{V}$ test, $u=1.38, P>.06$; fig. $4 F$ ). The angular-angular correlation between two consecutive turning angles was negligible $\left(r_{\mathrm{aa}}=0.032, n=43\right.$, random $95 \% \mathrm{CI}=-0.067$ to 0.064$)$.

\section{Interday Analysis}

In this section, we analyze a data set of 1,318 radiolocations from 30 dispersing animals, with a sampling frequency of one location per day and animal.

Daily distances. At this temporal scale, dispersing lynx moved between 0.20 and $18.0 \mathrm{~km}$ per day. The inverse cumulative probability distribution of distances follows an exponential decay function (table 2). Correlation between the distances moved in consecutive days was low but significantly different from that expected under a null hypothesis of random selection of distances from the exponential distribution $(r=0.25, P<.0001,95 \% \mathrm{CI}=$ -0.11 to 0.11 ; calculated from 10,000 randomizations of the original data). The correlation between the distances moved in two vectors separated by 1 day (i.e., secondorder correlation) was not significant $(r=0.10, P=$
$.080,95 \% \mathrm{CI}=-0.13$ to 0.14$)$. There was no correlation between the number of cells of dispersal habitat surrounding the first location of the animal and the distance moved per day $\left(r^{2}=0.02, P=.65\right)$. The frequency of movements (i.e., two locations) in open habitat was significantly lower than at the hourly scale $\left(3.9 \%\right.$ vs. $9.6 \%, \chi_{1}^{2}=$ $17.83, P<.001)$.

The distance moved in dispersal habitat (overall model; natural $\log$ transformed, $F=2.71, \mathrm{df}=47,481, \quad P<$ $.0001)$ was affected by the sex of the animal $(F=5.76$, $\mathrm{df}=1,481, P<.017)$, the season $(F=4.01, \mathrm{df}=3,481$, $P<.0078$ ), and individuals (nested in model design, $F=2.08, \mathrm{df}=42,481, P<.0001)$. At this temporal scale, females moved shorter distances than males (1.76 \pm $1.49 \mathrm{~km}, n=189$, and $2.78 \pm 2.52 \mathrm{~km}, n=340$, respectively), while distances moved during the spring tended to be shorter. The average distance of the displacements in open habitat was $60.6 \%$ larger than in dispersal habitat (table 2). The small sample size only allowed us to test for the effect of sex on the distance moved, with males tending to move longer distances than females (table 2; $F=3.25, \mathrm{df}=1,25, P=.0834)$. Movements in the open habitat were significantly larger than those in dispersal habitat or mixed movements (Kruskal-Wallis ANOVA on ranks, $H=10.59, \mathrm{df}=2, P=.005$; Dunn's post hoc $Q>2.845, P<.05$ in all comparisons, including movements in open habitat). Finally, there was no effect of sex, season, individual, or time since the beginning of dispersal 


\section{Intraday: moving from dispersal habitat}


Intraday: moving from open habitat


\section{Interday}


Figure 4: Distributions of turning angles defined by two consecutive movement vectors. In $A-F$, movement vectors are defined by three consecutive radiolocations obtained in three consecutive hours of the same period of activity. $A-C$ represent when the second location of the animal is in dispersal habitat, surrounded by eight or seven cells of dispersal habitat $(A)$, six to three cells $(B)$, and two to zero cells $(C)$. $D-F$ represent when the second location of the animal is in open habitat and surrounded by more than three cells of dispersal habitat $(D)$, three to one cells $(E)$, and by zero cells $(F)$. In $G-I$, the two movement vectors are defined by three consecutive daily locations (Interday). $G$ represents all data, while $H$ and $I$ represent when the second location of the animal is in dispersal habitat surrounded by eight to six cells of dispersal habitat and five to zero cells of dispersal habitat, respectively. In all cases, concentric reference circles are located every five observations.

on the distance moved in mixed movements $(F=1.33$, $\mathrm{df}=31,103, P=.1462$ ).

Landscape effects on turning angles. Turning angles between movement vectors of 1 day were not significantly oriented toward $180^{\circ}$ (fig. $4 G$; Rayleigh $V$-test, $u=1.21$, $P>.05)$ and were not different from a uniform distribution (Rayleigh $z=0.73, P>.20$ ). There was no effect of sex, season, and time since the beginning of dispersal on the cosine of the angles $(F<0.31, \mathrm{df}=28,281, P>$ .82 in all cases), while individuals were significant in the model (nested in model design $F=1.67, \mathrm{df}=28,281$, $P=.0214)$. In the majority of the turning angles, the sec- ond location of the animal was in dispersal habitat and mostly surrounded by dispersal habitat (six to eight cells of dispersal habitat). In this case, the distribution of turning angles was not different from uniform (fig. $4 \mathrm{H}$; Rayleigh $z=0.12, P>.50$ ). However, when the animals were in dispersal habitat and surrounded by fewer than seven cells of dispersal habitat, the movement was significantly oriented toward $180^{\circ}$ (fig. 4I; Rayleigh $V$-test, $u=2.53$, $P<.01)$. In the few cases in which lynx were inside open habitat, the distribution of turning angles was not different from uniform (Rayleigh $z=1.69, P>.1, n=25$ ), including when there were fewer than seven cells of dispersal 
habitat around the animal (Rayleigh $z=1.08, P>.2$, $n=19)$. There was no correlation between two consecutive turning angles $\left(r_{\mathrm{aa}}=0.00, \mathrm{CI}=-0.01\right.$ to 0.01$)$.

\section{The Rules of the Individual-Based Simulation Model}

Given the very large movement capacity of the lynx and the relatively small study area, we have to use a finegrained resolution in time and space. We have to define the movement capacity of individual lynx, the autocorrelation in their movement, and the habitat selection within the matrix. We assume that the animals can identify the type of habitat of their eight-cell neighborhood (i.e., a perceptual range of one cell). An animal performs a sequence of movement steps in which it can move to one of the surrounding eight cells or stay in its current position. However, it cannot move into barrier habitat. Individuals move independently of each other without competing for space.

Rule 1: Movement Capacity. The duration of a dispersal event is given by the number of days that a given individual moved. We stochastically determine the number of steps, $\phi_{\mathrm{d}}$, a lynx moves during a given day, sampling the accumulated density probability function of the total distance moved per period of activity (so that $p_{\mathrm{a}}[0$ steps $]=1$ and $p\left[\phi_{\mathrm{d}}\right]=p_{\mathrm{a}}\left[\phi_{\mathrm{d}}\right]-p_{\mathrm{a}}\left[\phi_{\mathrm{d}}+1\right]$; see table 2$)$ :

$$
p_{\mathrm{a}}\left(\phi_{\mathrm{d}}\right)=\left(1+a \cdot \phi_{\mathrm{d}}^{3}\right)^{-1},
$$

where $p_{\mathrm{a}}$ is the accumulated probability obtained and $a$ is the parameter that determines the movement capacity. For model analysis, we use the mean dispersal distance moved per day $\bar{\phi}_{\mathrm{d}}$, which is directly related to $a$ as a model parameter because $\bar{\phi}_{\mathrm{d}}$ has a more straightforward interpretation. Following a parsimonious model development, we did not include a sex-based difference in the movement capacity because with the data available we did not detect it at the intraday scale (even when it was present at the daily scale). In any case, if such a difference exists in the Iberian lynx, not including it in the model goes against our hypothesis of an appropriate model performance (we then would expect a reduced applicability of the model).

Rule 2: Habitat Selection-Moving to Open Habitat. Lynx selected cells of open habitat with the same probability as dispersal habitat if their actual location was surrounded by more than five cells of dispersal habitat, but they avoided moving to them if they were surrounded by fewer cells of dispersal habitat (see fig. 4). Therefore, we introduce a parameter $N_{\mathrm{d}}(=5)$ that defines the threshold below which the lynx perceive its surroundings as fragmented, and we model the avoidance of the matrix within fragmented areas (i.e., for $D<N_{\mathrm{d}}$ ) as

$$
\begin{aligned}
& P_{\mathrm{o}}(O, D)=\frac{1}{(O+D)} \beta, \\
& P_{\mathrm{d}}(O, D)=\frac{1-\left(O P_{\mathrm{o}}(O, D)\right)}{D},
\end{aligned}
$$

where $P_{\mathrm{o}}(O, D)$ and $P_{\mathrm{d}}(O, D)$ are the probabilities for a given cell of open and dispersal habitats, respectively, to be selected; $O$ and $D$ are the number of cells of open and dispersal habitats surrounding the location of the animal; and $\beta$ is the parameter describing the avoidance of open habitat (for $\beta=1$; no avoidance because $P_{\mathrm{o}}(O, D)=$ $\left.P_{\mathrm{d}}(O, D)=1 /(O+D)\right)$. Note that we explicitly consider $O$ and $D$ instead of the total number of cells because, by definition, cells of barrier habitat cannot be used, and $O P_{\mathrm{o}}(O, D)+D P_{\mathrm{d}}(O, D)=1$.

Rule 3: Habitat Selection-Moving Back to Dispersal Habitat. Once in open habitat, the lynx showed an increased accumulated probability to return to dispersal habitat as the period of activity passed. It was rare for them to remain in open habitat during the resting period; only $8 \%$ of the periods of activity that began in dispersal habitat ended in open habitat. We considered this movement property of the probability $P_{\mathrm{d}}$ to move toward the last cell of dispersal habitat visited in the current period of activity as a function of time:

$$
P_{\mathrm{d}}(T)=T \gamma
$$

where $\gamma$ is the parameter describing the return to dispersal habitat. The number of hours already moved during the current period of activity, $T$, was calculated as

$$
T=10.5 \frac{\phi_{\mathrm{c}}}{\phi_{\mathrm{d}}}
$$

where $\phi_{c}$ is the current step, 10.5 is the average duration of a period of activity (Palomares et al. 2001), and $\phi_{\mathrm{d}}$ is the total number of steps of the current day (stochastically determined after eq. [1]). Once headed toward the last cell of dispersal habitat, the individual moves in this direction until it detects a cell of dispersal habitat, and then it moves directly to it. In this way, lynx may explore the open habitat during their periods of activity, but they normally return to dispersal habitat as the period of activity passes.

Rule 4: Movement Autocorrelation. Intraday movements in unfragmented dispersal habitat (defined by $D \geq N_{d}$; see 
E142 The American Naturalist

Table 3: Sensitivity analysis of secondary model predictions (based on 25,000 random model parameterizations)

\begin{tabular}{|c|c|c|c|c|c|c|c|c|c|c|c|c|c|}
\hline \multirow[b]{2}{*}{ Prediction } & \multirow{2}{*}{$\begin{array}{c}\text { Observed } \\
\text { field } \\
\text { value }\end{array}$} & \multirow{2}{*}{$\begin{array}{l}\text { Simulated } \\
\text { minimum }\end{array}$} & \multirow{2}{*}{$\begin{array}{l}\text { Simulated } \\
\text { maximum }\end{array}$} & \multirow[b]{2}{*}{$r^{2}$} & \multicolumn{9}{|c|}{ Sensitivity coefficient $S$ for model parameters } \\
\hline & & & & & $\bar{\phi}_{d}$ & $\theta_{d}$ & $N_{d}$ & $\beta$ & $\gamma$ & $L$ & $\Delta \theta_{l}$ & $\Delta \theta_{f}$ & $\delta$ \\
\hline$c r-r b$ & .231 & .063 & .670 & .90 & .18 & .24 & -.12 & .21 & -.37 & -.06 & .02 & .18 & .00 \\
\hline$c r-m a$ & .077 & .000 & .140 & .69 & .24 & .17 & .02 & .07 & -.13 & -.05 & .02 & .10 & -.01 \\
\hline$c r-a c$ & .154 & .092 & .631 & .90 & .30 & .23 & -.03 & .13 & -.26 & -.06 & .02 & .14 & -.03 \\
\hline cr-ha & .385 & .211 & .523 & .74 & .35 & .01 & .19 & -.07 & .09 & .00 & .00 & -.04 & -.07 \\
\hline cr-to & .300 & .045 & .359 & .78 & .39 & .12 & .03 & .08 & -.05 & -.04 & .02 & .05 & -.03 \\
\hline$c r-p u$ & .150 & .000 & .100 & .70 & .30 & .11 & .01 & .04 & -.12 & -.06 & .03 & .07 & -.01 \\
\hline cr-mo & .154 & .000 & .221 & .81 & .32 & .15 & .08 & .01 & -.07 & -.05 & .04 & .06 & -.04 \\
\hline$r b-c r$ & .077 & .020 & .477 & .86 & .18 & .19 & -.10 & .14 & -.35 & -.05 & .02 & .13 & .00 \\
\hline$r b-m a$ & .231 & .048 & .308 & .65 & .34 & .17 & .13 & -.03 & -.01 & -.06 & .03 & .10 & .00 \\
\hline$r b-a c$ & .538 & .449 & .805 & .80 & .41 & .12 & .13 & -.06 & .03 & -.04 & .03 & .00 & -.10 \\
\hline$r b-h a$ & .000 & .007 & .024 & .73 & .24 & .15 & -.02 & .09 & -.20 & -.04 & .02 & .08 & -.02 \\
\hline$r b-t o$ & .000 & .000 & .191 & .72 & .24 & .12 & -.01 & .07 & -.13 & -.05 & .02 & .07 & -.01 \\
\hline$r b-p u$ & .000 & .000 & .066 & .44 & .12 & .05 & .00 & .02 & -.07 & -.03 & .02 & .03 & .00 \\
\hline$r b-m o$ & .000 & .001 & .249 & .78 & .30 & .11 & .09 & -.02 & -.01 & -.04 & .03 & .03 & -.05 \\
\hline$m c r$ & 45.7 & 2.6 & 37.3 & .94 & .39 & .20 & .06 & .05 & -.14 & -.06 & .04 & .10 & -.05 \\
\hline$m r b$ & 32.1 & 23.1 & 36.5 & .92 & .41 & .19 & .09 & .00 & -.09 & -.06 & .04 & .07 & -.06 \\
\hline$e c r$ & 32.5 & 3.0 & 58.9 & .86 & .37 & .19 & .06 & .02 & -.10 & -.06 & .04 & .09 & -.05 \\
\hline$e r b$ & 20.0 & 29.3 & 59.7 & .72 & .35 & .11 & .09 & -.06 & .02 & -.04 & .03 & .02 & -.06 \\
\hline$h a b$ & .14 & .06 & .29 & .85 & .00 & .05 & -.23 & .29 & -.37 & .00 & .00 & .03 & .00 \\
\hline netd & 4.2 & 2.9 & 7.0 & .94 & .40 & .14 & .09 & .04 & -.10 & -.03 & .02 & .06 & -.07 \\
\hline$a v d_{30}$ & 18.1 & 13.2 & 31.8 & .92 & .40 & .19 & .06 & .02 & -.10 & -.06 & .04 & .08 & -.05 \\
\hline$a v d_{120}$ & 25.4 & 23.3 & 42.8 & .88 & .46 & .20 & .09 & -.01 & -.08 & -.07 & .04 & .08 & -.06 \\
\hline Sensitivity average & & & & & .30 & .15 & .08 & .07 & .13 & .05 & .03 & .07 & .04 \\
\hline
\end{tabular}

Note: Table shows the value of the pattern observed in the field as well as the minimum and maximum of the patterns for all the parameterizations (calculated as the average of 50 simulations for each parameterization), the $r^{2}$ of regression with the prediction as dependent variable and all model parameters as independent variables, and the resulting sensitivity coefficient $S$ for each model parameter. See table 1 for a description of all parameters. In the "Prediction" column, $c r-r b, c r-m a, c r-a c, c r-h a, c r-t o, c r-p u$, and $c r-m o$ are the connectivities between Coto del Rey subpopulation and Reserva Biologica, Marismillas, Acebuche, Hato Raton, Torrecuadros, Puebla, and Moguer subpopulations; $r b$ - $c r, r b$ - $m a, r b$ - $a c, r b$ - $h a, r b$-to, $r b$ - $p u$, and $r b$ - $m o$ are the connectivities between Reserva Biologica subpopulation and Coto del Rey, Marismillas, Acebuche, Hato Raton, Torrecuadros, Puebla, and Moguer subpopulations; $m r b$ and $m c r$ are the averages of the maximum dispersal distances reached by animals beginning dispersal from Reserva Biologica and Coto del Rey subpopulations, respectively; erb and ecr are the averages of the final dispersal distances reached by animals beginning dispersal from Reserva Biologica and Coto del Rey subpopulations, respectively; hab is the proportion of locations in open habitat matrix at the daily temporal resolution; netd is the average net distance moved between days; $a v d_{30}$ and $a v d_{120}$ are the average distances to the origin of dispersal at days 30 and 120.

"Rule 2") showed an overall degree of autocorrelation, $\theta_{\mathrm{d}}$, defined as the probability to move in the same direction as the previous movement step (note that there was no general directional preference during dispersal and that the tendency did not translate into further orders of magnitude such as between days).

The distribution of turning angles in unfragmented dispersal habitat was bimodal, with a higher probability of moving in the previous direction and moving back. We estimated the probability of moving back as a fraction of the probability of moving ahead, with

$$
\theta_{\text {back }}=\theta_{\mathrm{d}} \cdot \delta
$$

being the possible range of $\delta$ between 0 (no probability of moving back) and 1 (the same probability of moving back as moving ahead). Again, the remaining probability (of moving to any other cell) is calculated following $\theta_{\mathrm{d}}$ and $\theta_{\text {back }}$.

Animals moving in fragmented areas (i.e., when $D<$ $N_{\mathrm{d}}$; see "Rule 2") increased the autocorrelation of their movement as

$$
\theta_{\mathrm{t}}=\theta_{\mathrm{d}}+\Delta \theta_{\mathrm{f}}
$$

where $\Delta \theta_{\mathrm{f}}$ is the increase in autocorrelation and $\theta_{\mathrm{t}}$ is the total autocorrelation.

The overall level of autocorrelation of the movement path increased when animals moved longer distances $(>3$ $\left.\mathrm{km} \mathrm{h}^{-1}\right)$. Therefore, if $\phi_{\mathrm{d}} / 10.5>L$, then

$$
\theta_{1}=\theta_{\mathrm{t}}+\Delta \theta_{1}
$$

where $L$ is the threshold parameter defining long-distance 
movements (given by the number of steps per hour), $\theta_{t}$ is the total probability of keeping the previous direction, $\Delta \theta_{1}$ is the increase due to long-distance movement, and $\theta_{1}$ is the final autocorrelation observed in long-distance movements.

\section{Sensitivity Analysis}

All regression functions were significant $(P<.0001)$, explaining a large proportion of the variance of the predictions (between $44 \%$ and $94 \%$; table 3 ). Therefore, regression models including only main effects (i.e., without considering the interactions existing between parameters) yield reasonably simple approximations to the general behavior of the model in the $n$-dimensional parameter space that we explored (table 1).

The most important parameters were the mean number of steps per day, $\bar{\phi}_{\mathrm{d}}$, and the overall autocorrelation in dispersal habitat, $\theta_{\mathrm{d}}$ (table 3 ). The first parameter $\bar{\phi}_{\mathrm{d}}$ affected most predictions, except for the habitat selection on a daily scale (hab; table 3 ). The lower sensitivity values corresponded to the connectivities between subpopulations separated by open habitat ( $c r-r b, r b-c r$, and $r b-p u$; table 3; fig. 1). The influence of autocorrelation $\theta_{\mathrm{d}}$ was low only for the connectivities $c r-h a, r b-p u$, and also $h a b$. The probability to return to dispersal habitat $\gamma$ was the next most important parameter, mostly in relation to those predictions in which the animals had to cross open habitat (e.g., $c r-r b, r b-c r, c r-a c$; see fig. 1) and, as expected, in the habitat selection, hab. This sensitivity in the predictions related to matrix heterogeneity (such as connectivities between subpopulations separated by open habitat or by corridors of dispersal habitat; see fig. 1) was also evident for the other parameters involved in habitat selection (number of cells of dispersal habitat defining fragmentation, $N_{\mathrm{d}}$, avoidance of open habitat, $\beta$, and the increase in autocorrelation in fragmented areas, $\Delta \theta_{\mathrm{f}}$; table 3 ). All other parameters show low sensitivities for the majority of the predictions (table 3 ). Note that we can use these regression models to evaluate the effect of different degrees of model uncertainty on model predictions.

\section{Model Performance}

We used observed field parameter values (e.g., by sampling the observed distributions; table 1) to evaluate the capacity of the model to reproduce the movement properties at higher scales (patterns), namely at the interday scale (predictions on habitat selection, hab, and net distance moved per day, netd) and at the metapopulation level (predictions on the connectivities between subpopulations, the final and the maximum distances reached by the animals, and the average distance to the subpopulation of origin at days
30 and 120). These patterns were calculated using the same field data set on dispersing Iberian lynx.

Most of the observed patterns were remarkably well within the range predicted by the model (table 4; fig. 5). To evaluate the impact of matrix heterogeneity on the prediction capacity of the model, we repeated the same number of simulations with a basic model that did not consider habitat selection in the matrix (i.e., dispersal habitat $=$ open habitat $=$ barrier habitat). The basic model that included only the two most important parameters (the number of steps per day and an autocorrelation parameter, made equal to the value of $\theta_{\mathrm{d}}$; see "Sensitivity Analysis") was also parameterized with the field data. The performance of the basic model was clearly reduced in comparison with the full model that contained habitat selection, especially for predictions in which we expect a constraining structure of the matrix habitats (e.g., $c r-r b$; table 4; fig. 5). The only improvement of the basic model against the full model was for the connectivity between subpopulations Coto del Rey and Puebla, cr-pu (table 4; fig. 5). The overall behavior of both models in their simulated predictions was considerably different (MANOVA, Wilks's $\lambda=0.005, F=19,049$, df $=22,1977, P<.0001)$.

The improvement of the full model over the basic model in connectivity values is shown also by the overall correlation between the values observed in the field and the simulated averages $(0.90[P<.0001]$ and $.57[P=.032]$ for the full and the basic models, respectively). Because the basic model represents only the movement capacity of lynx, while the effect of matrix heterogeneity is included in the full model, we find that the movement capacity explains $27 \%$ of the variance of field connectivities, while matrix heterogeneity explains an additional 53\% of the variance (i.e., the full model explains $80 \%$ of the variance in field connectivity values).

In the basic model, we assume a homogeneous matrix, and therefore we assume that connectivity between two given patches follows an exponentially declining colonization probability of the form

$$
\Gamma_{i j}=e^{-a d_{i j}}
$$

where $\Gamma_{\mathrm{ij}}$ represents the connectivity between patches $i$ and $j, d_{i j}$ is the distance between both patches, and $a$ is a scale parameter that sets the distance-dependent dispersal rate (e.g., Hanski 1994, 1999; Vos et al. 2001; Cronin 2003). In order to evaluate this general model (eq. [8]) where dispersing animals have to move in a heterogeneous matrix, we regressed for each source subpopulation (Coto del Rey and Reserva Biologica) the values of the connectivities estimated with the full model (i.e., recognizing matrix heterogeneity) and the field values against the values predicted by equation (8) (fig. 6). In both cases the fit was very poor, 
especially when compared with the connectivity values predicted by the basic model in which interpatch distance explains most of the variance (fig. 6). Therefore, the approximation of equation (8) to estimate interpatch connectivities in a metapopulation with heterogeneous matrix can be misleading, especially for close patches separated by open habitat or corridors (fig. 6).

\section{Discussion}

In fragmented landscapes, dispersal is the process of population dynamics that keeps local populations together, and our ability to manage and conserve species in fragmented landscapes depends critically on our understanding of the dispersal process and the way we conceptualize and model it. However, effects of matrix heterogeneity on dispersal are hard to model in a simple and realistic manner (e.g., Gustafson and Gardner 1996) and are believed to be extremely difficult to quantify and demonstrate in empirical studies (e.g., Moilanen and Hanski 1998). As a consequence of the scarcity of landscape-level empirical information on dispersal, theoretical and applied models are frequently based on untested assumptions of dispersal movement such as correlated random walks on a homogeneous matrix. In this article, we analyzed and modeled the dispersal movement of Iberian lynx, a species with complex cognitive capacities, within a metapopulation to reevaluate the current standard of plausibility in modeling dispersal movement. We analyzed the telemetric data on dispersing lynx to find out whether (and how) a heterogeneous matrix impacted movement properties. Next we constructed an individual-based spatially explicit dispersal model with movement rules derived from an analysis at the hour-to-hour temporal scale to investigate whether a model constructed with low-level information would be able to correctly predict high-level population parameters at the individual and population levels (e.g., connectivity, dispersal distance) and which movement properties are important for this.

\section{Movement Properties in a Heterogeneous Matrix}

We followed a behavioral approach (e.g., Lima and Zollner 1996; Haddad 1999b; Holway and Suarez 1999) to extract a set of behavioral movement properties from dispersal data. These properties describe the movement within different types of matrix and the response of the individuals to the spatial arrangement of habitat types in their surroundings when they take a movement decision. Before conducting the actual analyses of movement paths, we defined two important constraints. The first was the definition of the composition of the landscape with respect to the habitat selection of the species during dispersal (by defining barrier, open, and dispersal matrix habitat types), and the second was the definition of the spatiotemporal scales of the analyses and the determination of the approximate temporal scale at which individuals interact with landscape structure.

The analyses at the intraday and daily scales revealed several patterns in the behavioral responses to matrix heterogeneity, with the amount of dispersal habitat surrounding the position of the animal having a strong effect on movement properties. Lynx avoided moving into open habitat if the matrix was fragmented, but otherwise they did not hesitate to explore open habitat during the same period of activity. However, the probability of staying in open habitat for more than one period of activity was very low (as we can expect because we defined open habitat as the land use types avoided at the daily temporal scale; Palomares et al. 2000). Despite their low frequency, displacements in the open habitat are very important because they may influence movement rates between subpopulations separated by open habitat. This is especially important when corridors of dispersal habitat have gaps of open habitat (Beier and Noss 1998) or when there are steppingstones of dispersal habitat. In this way, the functional connectivity (Metzger and Décamps 1996; Wiegand et al. 1999; With et al. 1999; Ferreras 2001), and the final distance reached by dispersing individuals in fragmented landscapes (Hanski 1999) is also affected by the spatial configuration of avoided matrix.

The amount of dispersal habitat surrounding the position of the animal also influenced the direction of the movement and modified the degree of autocorrelation. During their hourly movements within unfragmented dispersal habitat, lynx tended to keep the same movement direction. However, at the daily resolution, any directionality was lost. In more fragmented areas, lynx increased the degree of autocorrelation of their movement paths. This increase can be interpreted as a way of leaving and/ or as a way to explore the lower quality area during the same period of activity (usually during one night). In very fragmented areas (very few cells of dispersal habitat) as well as when animals were in open habitat far from dispersal habitat, movement directionality was not different from random. These results indicate that the overall movement autocorrelation is less important than the degree of matrix heterogeneity, and its maintenance depends on the availability of an adequate matrix composition. The outcome of this interaction of individuals with the matrix during their periods of activity when moving in fragmented areas translated at the daily resolution into a tendency to move backward, thus showing a different pattern in movement properties at a broader temporal scale. 


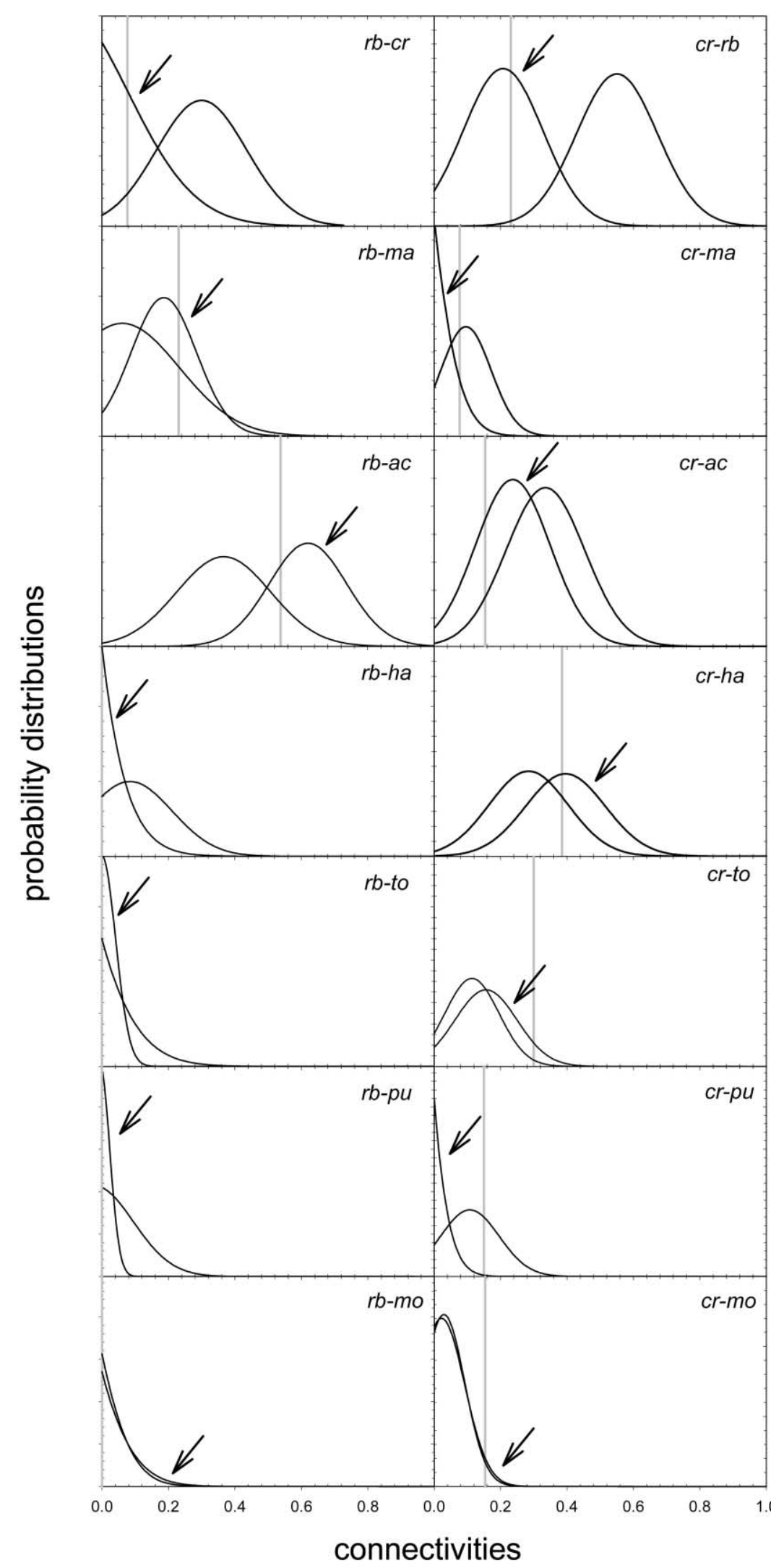

Figure 5 


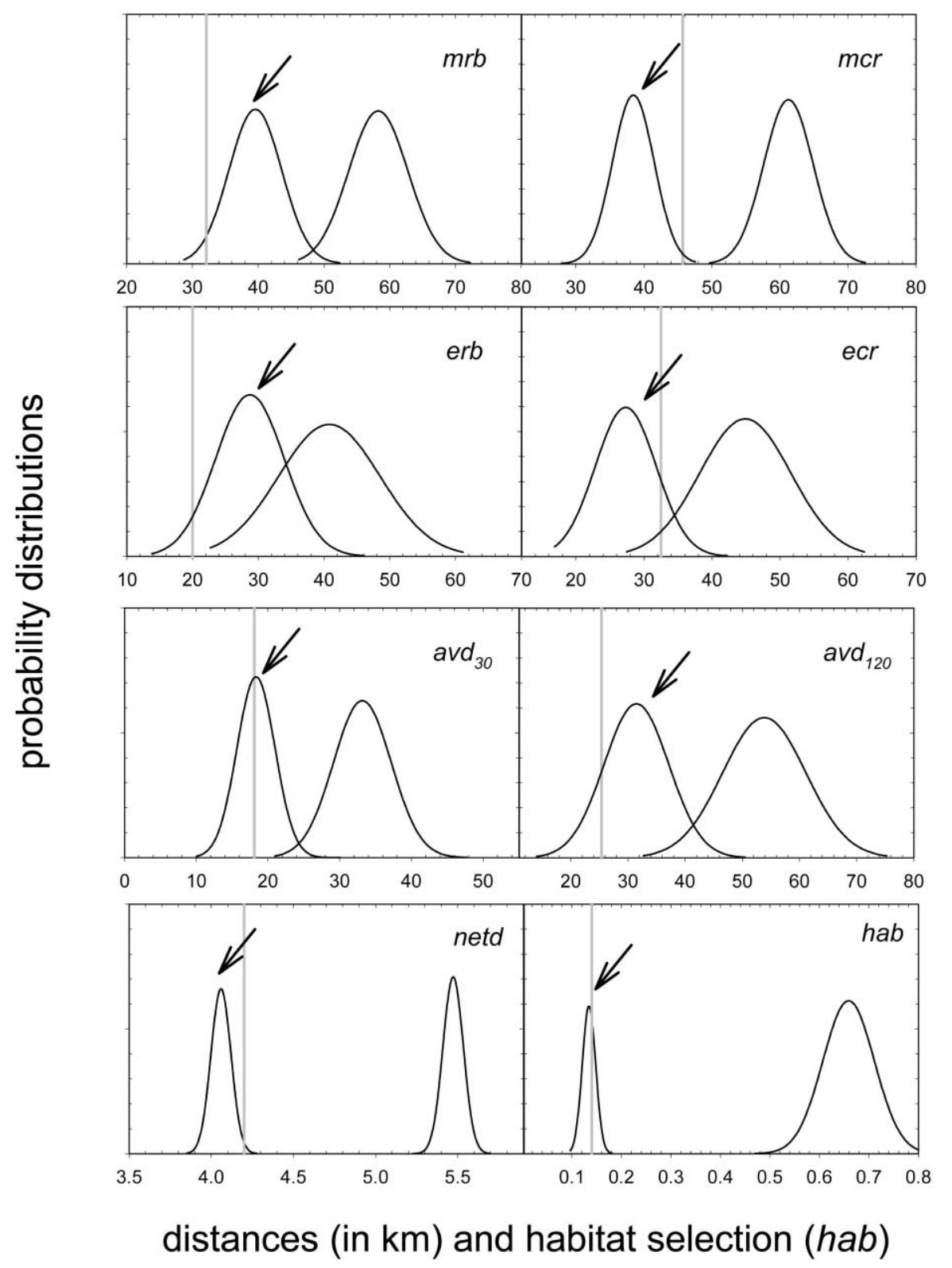

Figure 5: Distributions of the predictions made by two simulation models, one including rules on habitat selection during movements in the matrix or full model (distribution marked with an arrow) and one model including only the basic parameters on movement capacity or basic model. Vertical gray lines represent the values measured in the field. For a description of model predictions, see tables 3, 4.

\section{Modeling Individual Movement Behavior}

One of the most difficult parts of modeling dispersal is the estimation of the movement capacity of a species (Koenig et al. 1996), which is usually directly translated into a maximal number of steps moved (e.g., Pulliam et al. 1992; Ruckelshaus et al. 1997; Tyre et al. 1999; Wiegand et al. 1999). In our analyses, we found that the distri- butions of distances moved followed exponential decay functions. The usefulness of such distributions in the estimation (or prediction) of the movement capacity of a species is hampered by measurement errors and by the effect of the demographic and landscape frames on the distribution (e.g., Porter and Dooley 1993; Higgins and Richardson 1999; Clark et al. 2001). The latter effects are much more accentuated at broader temporal scales, es- 


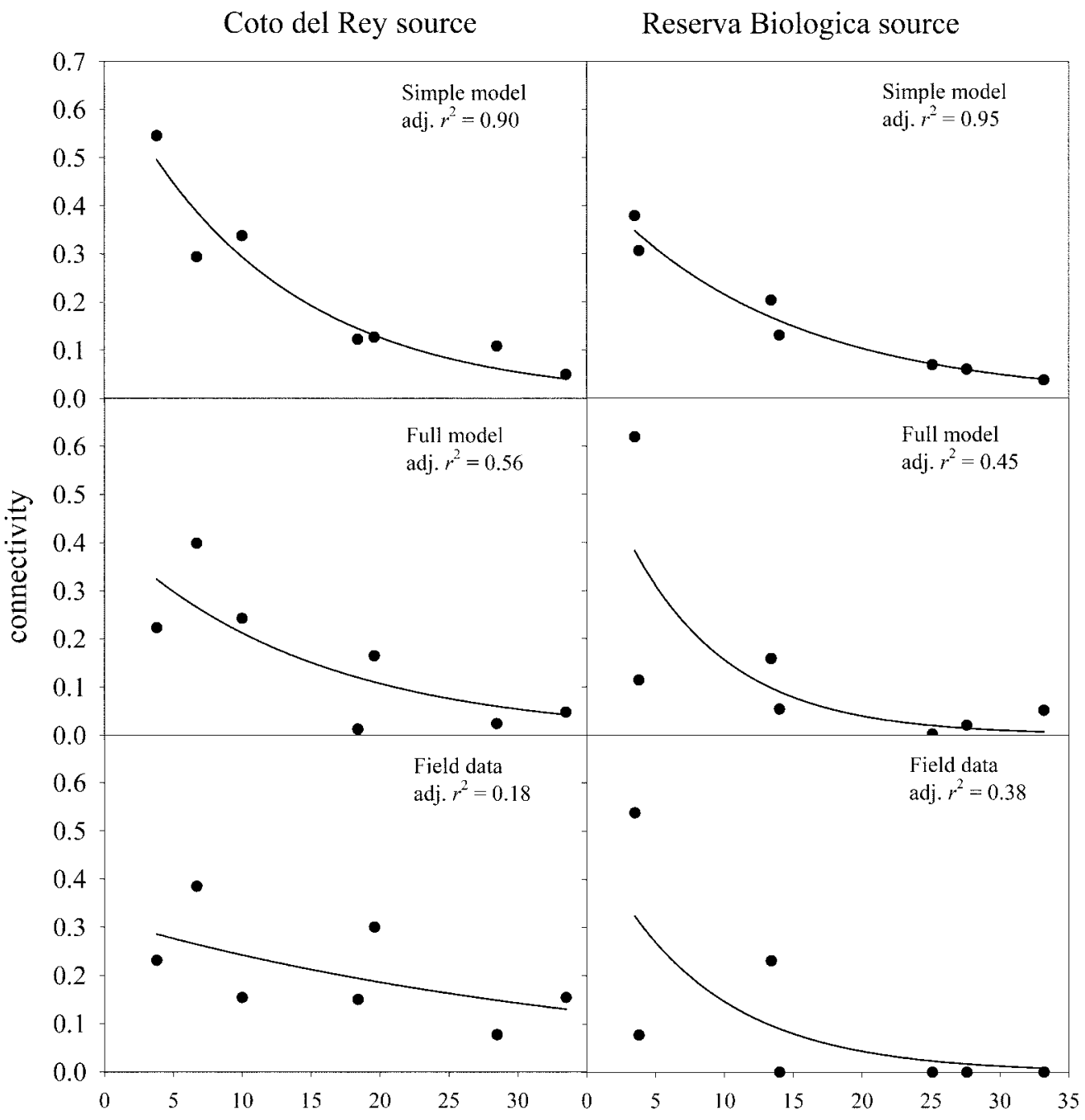

distance

Figure 6: Relationship between the connectivities from Coto del Rey and Reserva Biologica source populations and the rest of the subpopulations and interpatch distance following an exponential decay function. Connectivity values were estimated from a simulation model that included only rules on movement capacity (simple model), from a model that also included rules on habitat selection in the matrix (full model), and from field estimates. Only the predictions of the simple model significantly followed the exponential decay function.

pecially for maximum dispersal distances, which are also the most commonly reported. Following a more mechanistic approach, we used an alternative way to estimate movement capacity. Instead of using the total dispersal distance moved, we introduced a clearer temporal frame, that is, the period of activity. The estimation of the distribution of total distances moved per period of activity is easier and faster to obtain than that of maximum dispersal distances. This distribution of total distances can then be resampled for each simulated period of activity, and the model will deliver an estimate of the distance that one individual is expected to move during the duration of its dispersal period (in our case, expressed in number of days). In this way, even with a small number of marked animals tracked during only part of their dispersal period, we can obtain a realistic idea of the movement capacity of a species. The critical importance of a good estimation of the movement capacity was outlined by the fact that the mean number of steps moved per day was the most influential parameter of the simulation model, widely affecting model predictions.

The next most influential parameter was the overall au- 
Table 4: Comparison between a simulation model containing all movement rules on habitat selection within the matrix (full model) and another simulation model without them (basic model)

\begin{tabular}{|c|c|c|c|c|c|c|c|c|}
\hline \multirow{2}{*}{$\begin{array}{l}\text { Predicted } \\
\text { pattern }\end{array}$} & \multirow[b]{2}{*}{ Observed } & \multicolumn{3}{|c|}{ Simulated full model } & \multicolumn{3}{|c|}{ Simulated basic model } & \multirow{2}{*}{$\frac{\begin{array}{c}\text { Model } \\
\text { comparison }\end{array}}{r^{2}}$} \\
\hline & & Mean & $\mathrm{SD}$ & $P$ & Mean & SD & $P$ & \\
\hline$c r-r b$ & .231 & .223 & .116 & $>.5$ & .554 & .122 & $<.01$ & .65 \\
\hline$c r-m a$ & .077 & .024 & .046 & $>.2$ & .108 & .074 & $>.5$ & .33 \\
\hline$c r-a c$ & .154 & .242 & .109 & $>.5$ & .338 & .114 & $>.2$ & .15 \\
\hline cr-ha & .385 & .399 & .123 & $>.5$ & .294 & .114 & $>.2$ & .16 \\
\hline$c r$-to & .300 & .164 & .091 & $>.1$ & .127 & .080 & $<.05$ & .05 \\
\hline$c r-p u$ & .150 & .012 & .030 & $<.001$ & .123 & .083 & $>.5$ & .44 \\
\hline cr-mo & .154 & .048 & .050 & $>.05$ & .049 & .052 & $<.05$ & .00 \\
\hline$r b-c r$ & .077 & .114 & .098 & $>.5$ & .307 & .133 & $>.1$ & .40 \\
\hline$r b-m a$ & .231 & .158 & .117 & $>.5$ & .204 & .108 & $>.5$ & .04 \\
\hline$r b-a c$ & .538 & .619 & .119 & $>.5$ & .379 & .134 & $>.2$ & .48 \\
\hline$r b-h a$ & .000 & .054 & .073 & $>.2$ & .131 & .094 & $>.1$ & .17 \\
\hline$r b$-to & .000 & .020 & .043 & $>.5$ & .060 & .068 & $>.2$ & .11 \\
\hline$r b-p u$ & .000 & .002 & .013 & $>.5$ & .069 & .071 & $>.2$ & .30 \\
\hline$r b-m o$ & .000 & .051 & .064 & $>.2$ & .038 & .054 & $>.2$ & .01 \\
\hline$m c r$ & 45.7 & 38.5 & 3.0 & $<.02$ & 61.3 & 3.7 & $<.0001$ & .92 \\
\hline$m r b$ & 32.1 & 39.7 & 4.0 & $>.1$ & 58.4 & 4.3 & $<.0001$ & .84 \\
\hline$e c r$ & 32.5 & 27.4 & 4.5 & $>.2$ & 45.0 & 6.5 & $>.1$ & .71 \\
\hline$e r b$ & 20.0 & 28.8 & 5.2 & $>.1$ & 41.1 & 7.4 & $<.005$ & .48 \\
\hline$h a b$ & .14 & .135 & .013 & $>.5$ & .657 & .049 & $<.0001$ & .98 \\
\hline netd & 4.2 & 4.06 & .06 & $<.05$ & 5.47 & .06 & $<.0001$ & .99 \\
\hline$a v d_{30}$ & 18.1 & 18.5 & 2.6 & $>.5$ & 33.3 & 4.1 & $<.0001$ & .83 \\
\hline$a v d_{120}$ & 25.4 & 31.6 & 5.5 & $>.2$ & 53.8 & 7.0 & $<.0001$ & .76 \\
\hline
\end{tabular}

Note: Both models were parameterized with field data, were run for 1,000 simulations (i.e., sample size in the comparisons $n=2,000)$, and have in common the parameters determining movement capacity ( $\alpha$ and $\theta_{d}$; see "Results" for more details). See table 1 for a description of all parameters. Observed field values for connectivities are after Ferreras (2001), dispersal distances are after Ferreras et al. (2004), hab is after Palomares et al. (2000), and $a v d_{30}$ and $a v d_{120}$ are after E. Revilla, T. Wiegand, F. Palomares, P. Ferreras, and M. Delibes (unpublished data). $P$ = probability to obtain the observed field value from the full and the basic simulation models. $r^{2}=$ coefficient of determination after the MANOVA comparison of the full and the basic simulation models for each of the predictions.

tocorrelation observed in the displacement in dispersal habitat. A strong directionality in the movement is one of the properties most frequently stated when modeling individual dispersal (with autocorrelation values of 0.5 or higher; e.g., Schippers et al. 1996; McCarthy 1997; Letcher et al. 1998; Zollner and Lima 1999), thus producing straight dispersal paths (Zollner and Lima 1999). If we directly observe a moving animal, we can perceive such directionality in the path it follows. However, the question is whether such autocorrelation on small scales persists when the movement path is resampled at coarser temporal scales. For the Iberian lynx, we found a low degree of autocorrelation during periods of activity (in comparison with the values used in the literature) and no autocorrelation at the daily scale. Therefore, it is important to acknowledge that autocorrelation is a scale-dependent property and that we have to define explicitly the temporal scale at which it is introduced into a simulation model.
Our results give rise to a reevaluation of the standard of plausibility (Lima and Zollner 1996) regarding the temporal scales in modeling dispersal. Although time is usually considered in defining the sequence of demographic processes, it is usually neglected when modeling animal dispersal movements. A correct estimation of movement capacity and autocorrelation during dispersal requires the prior definition of the temporal scale at which individual movement is modeled. The lack of an explicit temporal dimension in individual-based simulation models has led to unfruitful discussions (e.g., Ruckelshaus et al. 1997; Mooij and DeAngelis 1999; South 1999) on error propagation introduced by the application of a per-step mortality rate. Field estimates of mortality rates have always had a clear temporal dimension (such as annual or seasonal mortality rates). Therefore, any attempt to analyze the impact of systematic errors in the field estimations of mortality rates on the performance of simulation models 
will be meaningless without accounting for the temporal dimension of the data entering the model.

Finally, predictions such as interpatch connectivities in which the source and the target subpopulations are separated by open habitat or connected by narrow corridors of dispersal habitat were strongly dependent on the parameters describing the effect of matrix heterogeneity on individual behavior. These findings are extremely important because they show that when matrix heterogeneity affects individual behavior, the connectivity between subpopulations is more affected by the habitat distribution in the landscape than by interpatch distance. In the case of the Iberian lynx, this interaction of individual behavior with the landscape results in asymmetric interpatch connectivities (Ferreras 2001). A further improvement in the representation of dispersal, therefore, is to include any potential impact of matrix heterogeneity on individual movement properties (Ricketts 2001).

\section{Translating Individual Behavior into Population-Level Parameters}

We found that we can use (low-level) movement properties as a mechanistic approximation of the dispersal process for predicting population level parameters (see also $\mathrm{Na}$ than et al. 2002). This result is far from being trivial because we assumed that the behavior of animals such as large mammals, which have complex cognitive capacities and search strategies, can be described as a set of simple rules at only one temporal scale (the intraday scale). Therefore, individual-based spatially explicit simulation models potentially can be a powerful tool in the study of the impact of landscape heterogeneity on population dynamics. As in any other model, the representation of dispersal is a simplification of complex processes, with a structure used to capture more detailed interactions by integrating over processes in order to derive descriptions of the behavior of the system at different scales (Levin and Pacala 1997). In this way, an inadequate description of the component ecological hypotheses will lead to an incorrect process representation and hence to a model with a very low performance at higher scales (Reynolds and Ford 1999). Therefore, the study of model performance at different scales (individual-daily and population-dispersal event) is important to assess whether we can use the model to study the impact of landscape structure on population dynamics or not.

In our model, a precondition for obtaining mechanistic movement rules is the approximate definition of the temporal scale at which the individuals interact with landscape structure. The estimation of the movement capacity of the animal and the explicit introduction of a temporal frame are the most important ingredients in modeling dispersal of the Iberian lynx. However, movement capacity alone explained only $27 \%$ of the variance of field connectivity values, while matrix heterogeneity explained an additional $53 \%$ of the variance. Therefore, the second important ingredient is to validate the assumption of a homogeneous matrix and in case of need, to introduce movement rules related with matrix heterogeneity.

Estimating population-level dispersal parameters such as the ones we used to evaluate model performance is a daunting task. Sample size is always limiting, affecting the quality of the data obtained and widening the uncertainty of the estimates. For example, the field estimation of all 56 connectivities between the subpopulations of lynx in Doñana would require the capture and tagging of numerous individuals in each of them. On the other hand, data on individual behavior is certainly more attainable; a less intense sampling effort is required to generate the behavioral movement rules that adequately describe individual dispersal movements. This is an important advantage of mechanistic movement models over the direct field estimation of population-level parameters.

\section{Matrix Heterogeneity and Interpatch Movement Rates}

Many metapopulation models approximate the population level outcome of individual lifetime dispersal events (i.e., patch connectivity) with functions that relate the movement capacity of the species with features of landscape structure (see review in Moilanen and Nieminen 2002). The matrix is assumed to be homogeneous, and the probability of interpatch movements depends on the distance between the source and the target subpopulations (eq. [8]). As expected from the results of our individual-based dispersal models, however, this functional relationship holds only for the predictions of the basic model, which considered only the movement capacity of the individuals (unbiased autocorrelated random walk) but no matrix heterogeneity. However, including realistic effects of matrix heterogeneity in the simulation model greatly reduced the predictive capacity of the simple patch connectivity model, thus indicating the importance of considering matrix heterogeneity when estimating interpatch connectivities in metapopulations.

Previous attempts by Moilanen and Hanski (1998) to introduce the effect of matrix heterogeneity on patch isolation by including weight factors to the distances $d_{i j}$ in equation (8) produced a poor improvement in the fit of the incidence function. The authors interpret the lack of improvement as a result of the deficient match between the spatial resolution of patch size and that of their GIS cartography and because of the assumption of a straight dispersal path between subpopulations to quantify matrix structure between patches. Therefore, one of the perhaps 
most challenging tasks in the future development of metapopulation theory is finding general patch connectivity measures that adequately describe the impact of matrix heterogeneity on the outcome of the dispersal process (Tischendorf and Fahrig 2000; Ovaskainen 2004) or, alternatively, to develop individual-based dispersal submodels for determining interpatch connectivities. Further research should focus on the impact of individual variability, the relationship between model complexity and performance, and on how the impact of matrix heterogeneity on individual behavior translates into metapopulation viability. These lines of research represent a promising tool for the conservation of the Iberian lynx and of similar species surviving in landscapes that are intensively transformed and used by humans (e.g., the design of optimal corridors and stepping-stones, or the identification of critical habitat patches for the conservation of a given population, even when those patches are not the key breeding habitat).

\section{Conclusion}

Matrix heterogeneity had important effects on dispersal movement properties of Iberian lynx at the individual scale, and these effects depend on the temporal resolution of data. The effect of matrix heterogeneity on the individual scale translated to large effects on the population scale. The existence of matrix heterogeneity has important consequences for modeling interpatch connectivities in metapopulation models. We found that a careful implementation of descriptive movement rules under the same spatiotemporal scales as the description of movement properties can provide a mechanistic approximation of the dispersal process and hence can be used to effectively predict population parameters such as connectivity values between subpopulations. A purely patch-centered approach seems to be an unrealistic framework to analyze population processes occurring in complex landscapes when the characteristics of the habitat matrix should be explicitly incorporated into the assessment of species' responses to habitat fragmentation (Brotons et al. 2003). A more realistic approach for modeling individual dispersal in real landscapes would include several levels of matrix quality that are based on the ecology of the species, that is, behavioral movement rules affected by habitat quality, within clear and biologically meaningful spatial and temporal scales. This approach is not necessarily more complex but will make the standard of plausibility more realistic in the implementation of mechanistic approaches for describing dispersal. These mechanistic approaches are essential for deriving generalizations about how spatial heterogeneity influences ecological systems (Wiens et al. 1993).

\section{Acknowledgments}

Administrations of Doñana National Park and the Consejería de Medio Ambiente of the Junta de Andalucía gave all the permissions needed to perform field work. This study was partially financed by the Spanish Comisión Asesora de Investigación Científica y Técnica, the Dirección General de Investigación Científica y Técnica, and the Dirección General de Investigación (project numbers 944, PB87-0405, PB90-1018, PB94-0480, PB97-1163, and BOS2001-2391-C02-01), by Consejería de Medio Ambiente of the Junta de Andalucía, and by the Spanish Ministry of Environment (formerly Instituto Nacional para la Conservación de la Naturaleza) and was sponsored by Land Rover España S.A. Funding provided by the Umweltforschungszentrums-Centre for Environmental Research, Leipzig, enabled authors to travel between Germany and Spain for cooperative work. E.R. was supported by a Marie Curie Individual Fellowship (EVK2-CT-199950001) and by a Ramón y Cajal Contract. J. J. Aldama, J. Ayala, J. F. Beltrán, J. Calzada, J. M. Fedriani, N. Fernández, R. Laffitte, and J.C. Rivilla trapped some of the animals and/or did some radio tracking. We thank J. Calzada, N. Fernández, S. Higgins, S. Schadt, and O. Schmitz for assistance during the development of ideas or for comments on drafts of the manuscript.

\section{Literature Cited}

Batschelet, E. 1981. Circular statistics in biology. Academic Press, London.

Beier, P., and R. F. Noss. 1998. Do habitat corridors provide connectivity? Conservation Biology 12:1241-1252.

Beissinger, S. R., and M. I. Westphal. 1998. On the use of demographic models of population viability in endangered species management. Journal of Wildlife Management 62:821-841.

Brachet, S., I. Olivieri, B. Godelle, E. Klein, N. FrascariaLacoste, and P.-H. Gouyon. 1999. Dispersal and metapopulation viability in a heterogeneous landscape. Journal of Theoretical Biology 198:479-495.

Brotons, L., M. Mönkkönen, and J. L. Martin. 2003. Are fragments islands? landscape context and density-area relationships in boreal forest birds. American Naturalist 162:343-357.

Cantrell, R. S., and C. Cosner. 1999. Diffusion models for population dynamics incorporating individual behaviour at boundaries: applications to refuge design. Theoretical Population Biology 55:189-207.

Clark, J. S., M. Lewis, and L. Horvath. 2001. Invasion by extremes: population spread with variation in dispersal and reproduction. American Naturalist 157:537-554.

Cronin, J. T. 2003. Movement and spatial population 
structure of a prairie planthopper. Ecology 84:11791188.

Delibes, M., A. Rodríguez, and P. Ferreras. 2000. Action plan for conservation of the Iberian lynx (Lynxpardinus) in Europe. Nature and the environment. Vol. 111. Council of Europe, Strasbourg, France.

Doak, D. F., and L. S. Mills. 1994. A useful role for theory in conservation. Ecology 75:615-626.

Eastman, J. R. 1995. IDRISI for Windows: user's guide. Clark Labs for Cartographic Technology and Geographic Analysis, Clark University, Worcester, Mass.

Fernández, N., M. Delibes, F. Palomares, and D. J. Mladenoff. 2003. Identifying breeding habitat for the Iberian lynx: inferences from a fine-scale spatial analysis. Ecological Applications 13:1310-1324.

Ferreras, P. 2001. Landscape structure and asymmetrical inter-patch connectivity in a metapopulation of the endangered Iberian lynx. Biological Conservation 100: 125-136.

Ferreras, P., P. Gaona, F. Palomares, and M. Delibes. 2001. Restore habitat or reduce mortality? implications from a population viability analysis of the Iberian lynx. Animal Conservation 4:265-274.

Ferreras, P., M. Delibes, F. Palomares, J. M. Fedriani, J. Calzada, and E. Revilla. 2004. Proximate and ultimate causes of dispersal in the Iberian lynx Lynx pardinus. Behavioral Ecology 15:31-40.

Fisher, J., and D. B. Lindenmayer. 2000. An assessment of the published results of animal relocations. Biological Conservation 96:1-11.

Gaona, P., P. Ferreras, and M. Delibes. 1998. Dynamics and viability of a metapopulation of the endangered Iberian lynx (Lynx pardinus). Ecological Monographs 68:349-370.

Gustafson, E. J., and R. H. Gardner. 1996. The effect of landscape heterogeneity on the probability of patch colonization. Ecology 77:94-107.

Haddad, N. M. 1999a. Corridor and distance effects on interpatch movements: a landscape experiment with butterflies. Ecological Applications 9:612-622.

- 1999b. Corridor use predicted from behaviors at habitat boundaries. American Naturalist 153:215-227.

Haddad, N. M., and K. A. Baum 1999. An experimental test of corridor effects on butterfly densities. Ecological Applications 9:623-633.

Hanski, I. 1994. A practical model of metapopulation dynamics. Journal of Animal Ecology 63:151-162.

- 1999. Habitat connectivity, habitat continuity, and metapopulations in dynamic landscapes. Oikos 87:209219.

Hartway C., M. Ruckelshaus, and P. Kareiva. 1998. The challenge of applying spatially explicit models to a world of sparse and messy data. Pages 215-223 in J. Bascompte and R. V. Solé, eds. Modeling spatiotemporal dynamics in ecology. Springer, Berlin.

Haynes, K. J., and J. T. Cronin. 2003. Matrix composition affects the spatial ecology of a prairie planthopper. Ecology 84:2856-2866.

Higgins, S. I., and D. M. Richardson. 1999. Predicting plant migration rates in a changing world: the role of longdistance dispersal. American Naturalist 153:464-475.

Holway, D. A., and A. V. Suarez. 1999. Animal behavior: an essential component of invasion biology. Trends in Ecology \& Evolution 14:328-330.

Kindvall, O. 1999. Dispersal in a metapopulation of the bush cricket, Metrioptera bicolor (Orthoptera: Tettigoniidae). Journal of Animal Ecology 68:172-185.

Koenig, W. D., D. van Vuren, and P. N. Hooge. 1996. Detectability, philopatry, and the distribution of dispersal distances in vertebrates. Trends in Ecology \& Evolution 11:514-517.

Letcher, B. H., J. A. Priddy, J. R. Walters, and L. B. Crowder. 1998. An individual-based, spatially explicit simulation model of the population dynamics of the endangered red-cockaded woodpecker, Picoides borealis. Biological Conservation 86:1-14.

Levin, S. A. 1992. The problem of pattern and scale in ecology. Ecology 73:1943-1967.

Levin, S. A., and S. W. Pacala. 1997. Theories of simplification and scaling of spatially distributed processes. Pages 271-295 in D. Tilman and P. Kareiva, eds. Spatial ecology: the role of space in population dynamics and interspecific interactions. Monographs in Population Biology. No. 30. Princeton University Press, Princeton, N.J.

Levins, R. 1970. Extinction. Pages 77-107 in M. Gerstenhaber, ed. Some mathematical problems in biology. American Mathematical Society, Providence, R.I.

Lima, S. L., and P. A. Zollner. 1996. Towards a behavioral ecology of ecological landscapes. Trends in Ecology \& Evolution 11:131-135.

Linnell, J. D. C., R. Aanes, J. E. Swenson, J. Odden, and M. E. Smith. 1997. Translocation of carnivores as a method for managing problem animals: a review. Biodiversity and Conservation 6:1245-1257.

McCarthy, M. A. 1997. Competition and dispersal from multiple nest. Ecology 78:873-883.

McCarthy, M. A., M. A. Burgman, and S. Ferson. 1995. Sensitivity analyses for models of population viability. Biological Conservation 73:93-100.

McIntyre, N. E., and J. A. Wiens. 1999. How does habitat patch size affect animal movement? an experiment with darkling beetles. Ecology 80:2261-2270.

Metzger, J.-P., and H. Décamps. 1996. The structural connectivity threshold: an hypothesis in conservation biology at the landscape scale. Acta Oecologica 18:1-12. 
Moilanen, A., and I. Hanski. 1998. Metapopulation dynamics: effects of habitat quality and landscape structure. Ecology 79:2503-2515.

- 2001. On the use of connectivity measures in spatial ecology. Oikos 95:147-151.

Moilanen, A., and M. Nieminen. 2002. Simple connectivity measures in spatial ecology. Ecology 83:1131-1145.

Mooij, W. M., and D. L. DeAngelis. 1999. Error propagation in spatially explicit population models: a reassessment. Conservation Biology 13:930-933.

- 2003. Uncertainty in spatially explicit animal dispersal models. Ecological Applications 13:794-805.

Moreira, J. M., and A. Fernández-Palacios. 1995. Usos y coberturas vegetales del suelo en Andalucía: seguimiento a través de imágenes de satélite. Junta de Andalucía, Consejería de Medio Ambiente.

Nathan, R., G. G. Katul, H. S. Horn, S. M. Thomas, R. Oren, R. Avissar, S. W. Pacala, and S. A. Levin. 2002. Mechanisms of long-distance dispersal of seeds by wind. Nature 418:409-413.

Nathan, R., G. Perry, J. T. Cronin, A. E. Strand, and M. L. Cain. 2003. Methods for estimating long-distance dispersal. Oikos 103:261-273.

Norton, M. R., S. J. Hannon, and F. K. A. Schmiegelow. 2000. Fragments are not islands: patch vs. landscape perspectives on songbird presence and abundance in a harvested boreal forest. Ecography 23:209-223.

Ovaskainen, O. 2004. Habitat-specific movement parameters estimated using mark-recapture data and a diffusion model. Ecology 85:242-257.

Pagel, M., and R. J. H. Payne. 1996. How migration rates affect estimation of extinction threshold. Oikos 76:323329.

Palomares, F. 2001. Vegetation structure and prey abundance requirements of the Iberian lynx: implications for the design of reserves and corridors. Journal of Applied Ecology 38:9-18.

Palomares, F., M. Delibes, P. Ferreras, J. M. Fedriani, J. Calzada, and E. Revilla. 2000. Iberian lynx in a fragmented landscape: pre-dispersal, dispersal and postdispersal habitats. Conservation Biology 14:809-818.

Palomares, F., M. Delibes, E. Revilla, J. Calzada, and J. M. Fedriani. 2001. Spatial ecology of Iberian lynx and abundance of European rabbits in southwestern Spain. Wildlife Monographs 148:1-36.

Palomares, F., M. Delibes, P. Ferreras, J. Aldama, E. Revilla, J. Calzada, and N. Fernández. 2003. Estructura de la metapoblación de linces de Doñana. Pages 505-526 in J. M. Pérez Jiménez, ed. In memoriam al Prof. Dr. Isidoro Ruiz Martínez. Servicio de Publicaciones Universidad de Jaén, Spain.

Perault, D. R., and M. V. Lomolino. 2000. Corridors and mammal community structure across a fragmented, old- growth forest landscape. Ecological Monographs 70: 401-422.

Porter, J. H., and J. L. Dooley. 1993. Animal dispersal patterns: a reassessment of simple mathematical models. Ecology 74:2436-2443.

Pulliam, H. R., and J. B. Dunning. 1995. Spatially explicit population models. Ecological Applications 5:2.

Pulliam, H. R., J. B. Dunning, and J. Liu. 1992. Population dynamics in complex landscapes: a case study. Ecological Applications 2:165-177.

Reynolds, J. H., and E. D. Ford. 1999. Multi-criteria assessment of ecological process models. Ecology 80:538553.

Ricketts, T. H. 2001. The matrix matters: effective isolation in fragmented landscapes. American Naturalist 158:8799.

Ruckelshaus, M., C. Hartway, and P. Kareiva. 1997. Assessing the data requirements of spatially explicit dispersal models. Conservation Biology 11:1298-1306.

Schippers, P., J. Verboom, J. P. Knaapen, and R. C. van Apeldoorn. 1996. Dispersal and habitat connectivity in complex heterogeneus landscapes: an analysis with a GIS-based random walk model. Ecography 19:97-106.

South, A. 1999. Dispersal in spatially explicit population models. Conservation Biology 13:1039-1046.

Thomas, C. 2000. Dispersal and extinction in fragmented landscapes. Proceedings of the Royal Society of London B 267:139-145.

Tischendorf, L., and L. Fahrig. 2000. On the usage and measurement of landscape connectivity. Oikos 90:7-19.

Turchin, P. 1996. Fractal analyses of animal movement: a critique. Ecology 77:2086-2090.

- 1998. Quantitative analysis of movement: measuring and modeling population redistribution in animals and plants. Sinauer, Sunderland, Mass.

Tyre, A. J., H. P. Possingham, and D. B. Lindenmayer. 1999. Modelling dispersal behaviour on a fractal landscape. Environmental Modelling and Software 14:103113.

Vandermeer, J., and R. Carvajal. 2001. Metapopulation dynamics and the quality of the matrix. American Naturalist 158:211-220.

Vos, C. C., J. Verboom, P. F. M. Opdam, and C. J. F. Ter Braak. 2001. Toward ecologically scaled landscape indices. American Naturalist 183:24-41.

Wiegand, T., K. A. Moloney, J. Naves, and F. Knauer. 1999. Finding the missing link between landscape structure and population dynamics: a spatially explicit perspective. American Naturalist 154:605-627.

Wiegand, T., F. Jeltsch, I. Hanski, and V. Grimm. 2003. Using pattern-oriented modeling for revealing hidden information: a key for reconciling ecological theory and application. Oikos 100:209-222. 
Wiens, J. A., N. C. Stenseth, B. van Horne, and R. A. Ims. 1993. Ecological mechanisms and landscape ecology. Oikos 66:369-380.

With, K. A. 1994. Ontogenetic shifts in how grasshoppers interact with landscape patterns. Functional Ecology 8: 477-485.

With, K. A., S. J. Cadaret, and C. Davis. 1999. Movement responses to patch structure in experimental fractal landscapes. Ecology 80:1340-1353.
Zar, J. H. 1999. Biostatistical analysis. 4th ed. PrenticeHall, Upper Saddle River, N.J.

Zollner, P. A., and S. L. Lima. 1999. Search strategies for landscape-level interpatch movements. Ecology 80: 1019-1030. 